NOT FOR QUOTATION WITHOUT PERMISSION

OF THE AUTHOR

\begin{abstract}
DESIGNING APPROXIMATION SCHEMES FOR STOCHASTIC OPTIMIZATION PROBLEMS, IN PARTICULAR FOR

STOCHASTIC PROGRAMS WITH RECOURSE
\end{abstract}

John Birge

Roger J-B Wets

November 1983

WP-83-11]

Working Papers are interim reports on work of the International Institute for Applied Systems Analysis and have received only limited review. Views or opinions expressed herein do not necessarily represent those of the Institute or of its National Member organizations.

INTERNATIONAL INSTITUTE FOR APPLIED SYSTEMS ANALYSIS A-2361 Laxenburg, Austria 
PREFACE

The System and Decision Sciences Area has been involved in procedures for approximation as part of a variety of projects involving uncertainties. In this paper, the authors discuss approximation methods for stochastic programming problems. This is especially relevant to the Adaptation and Optimization project since it directly applies to the solution of optimization problems under uncertainty.

Andrzej P. Wierzbicki Chairman

System and Decision

Sciences Area 


\section{DESIGNING APPROXIMATION SCHEMES FOR \\ STOCHASTIC OPTIMIZATION PROBLEMS, \\ IN PARTICULAR FOR \\ STOCHASTIC PROGRAMS WITH RECOURSE}

John Birge

Industrial and Operations Engineering University of Michigan and

Roger $J-B$. Wets Department of Mathematics University of Kentucky

\section{$\underline{\text { ABSTRACT }}$}

Various approximation schemes for stochastic optimization problems involving either approximates of the probability measures and/or approximates of the objective functional, are investigated. We discuss their potential implementation as part of general procedures for solving stochastic programs with recourse.

Supported in part by grants of the National Science Foundation. 


\section{INTRODUCTION}

We take

$$
\text { find } x \in R^{n} \text { that minimizes } E_{f}(x)=E\{f(x, \xi(\omega))\}
$$

as prototype for the class of stochastic optimization problems under investigation, where

$$
\text { (1.2) } E\{f(x, \xi(\omega))\}=\int f(x, \xi(\omega)) P(d \omega),
$$

$\xi$ is a random vector which maps the probability space $(\Omega, A, P)$ on $\left(R^{N}, B^{N}, F\right)$ with $F$ the distribution function and $\Xi \subset R^{N}$ the support of the probability measure induced by $\xi$ (i.e. $\Xi$ is the set of possible values assumed by $\xi$ ), and $f: R^{n} \times R^{N} \rightarrow R \cup\{+\infty\}$ is an extended real-valued function. We shall assume:

$$
\text { for all } x, \omega \leftrightarrow f(x, \xi(\omega)) \text { is measurable, }
$$

and the following integrability condition:

$$
\text { if } P[\omega \mid f(x, \xi(\omega))<+\infty]=1 \text { then } E_{f}(x)<+\infty \text {. }
$$

We refer to $\mathrm{E}_{\mathrm{f}}=\mathrm{E}\{\mathrm{f}(\cdot, \xi(\omega))\}$ as an expectation functional. Note that it can also be expressed as a Lebesgue-Stieltjes integral with respect to $F$ :

$$
E_{f}(x)=\int_{R}^{N} f(x, \zeta) d F(\zeta)
$$

A wide variety of stochastic optimization problems fit into this (abstract) framework; in particular stochastic programs with (fixed) recourse [1]

$$
\begin{aligned}
& \text { (1.6) find } x \in R_{+}^{n_{1}} \text { such that } A x=b \text {, } \\
& \text { and } z=c x+Q(x) \text { is minimized }
\end{aligned}
$$

where $A$ is an $m_{1} \times n_{1}$-matrix, $b \in R^{m_{1}}$,

(1.7) $\quad Q(x)=E\{Q(x, \xi(\omega))\}=\int Q(x, \xi(\omega)) P(d \omega)$,

and, the recourse function is defined by

(1.8) $Q(x, \xi(\omega))=\inf \underset{y \in R_{+}}{n_{2}}\{q(\omega) y \mid W y=h(\omega)-T(\omega) x\}$. 
The $\left(m_{2} \times n_{2}\right)$-matrix $W$ is called the recourse matrix. For each $\omega: T(\omega)$ is $m_{2} \times n_{1}$, $q(\omega) \in R^{n_{2}}$ and $h(\omega) \in R^{m_{2}}$. Piecing together the stochastic components of the problem, we get a vector $\xi \in R^{N}$ with $N=n_{2}+m_{2}+\left(m_{2} \times n_{1}\right)$, and

$$
\xi=\left(q_{1}, \ldots, q_{n_{2}}, h_{1}, \ldots, h_{m_{2}}, t_{11}, \ldots, t_{1 n_{1}}, t_{21}, \ldots, t_{m_{2}, n_{1}}\right) .
$$

We set

$$
f(x, \xi)=\left[\begin{array}{ll}
c x+Q(x, \xi) & \text { if } A x=b, x \geq 0, \\
+\infty & \text { otherwise. }
\end{array}\right.
$$

Provided the recourse problem is a.s. bounded, i.e.

$$
P[\omega \mid \exists \pi \text { such that } \pi W \leq q(\omega)]=1 \text {, }
$$

which we assume henceforth, the function $Q$ and thus also $f$, does not take on the value $-\infty$. The measurability of $f(x, \bullet)$ follow-directly from that of $\xi \leftrightarrow Q(x, \xi)$ [1, Section 3]. If $\xi$ has finite second moments, then $2(x)$ is finite whenever $\omega \leftrightarrow Q(x, \xi(\omega))$ is finite [1, Theorem 4.1] and this guarantees condition (1.4).

Much is known about problems of this type [1]. The properties of $f$ as defined through (1.9), quite often motivate and justify the conditions under which we obtain various results. The relevant properties are

(1.11) $(\mathrm{h}, \mathrm{T}) \mapsto \mathrm{Q}(\mathrm{x}, \xi=(\mathrm{q}, \mathrm{h}, \mathrm{T}))$ is a piecewise linear convex function

$$
\text { for all feasible } x \in K=K_{1} \cap K_{2} \text {, }
$$

where

$$
\begin{aligned}
& K_{1}=\{x \mid A x=b, \quad x \geq 0\} \\
& K_{2}=\{x \mid \forall \xi(\omega) \in \Xi, \exists y \geq 0 \text { such that } W y=h(\omega)-T(\omega) x\},
\end{aligned}
$$

(1.12) $\mathrm{q} \leftrightarrow \mathrm{Q}(\mathrm{x}, \xi=(\mathrm{q}, \mathrm{h}, \mathrm{T}))$ is a concave piecewise linear function, and

$$
\mathrm{x} \mapsto \mathrm{Q}(\mathrm{x}, \xi) \text { is a convex piecewise linear function }
$$

which implies that 

$x \mapsto Q(x)$ is a Lipschitzian convex function,

finite on $K_{2}$, as follows from the integrability condition on $\xi(\bullet)$.

When $T$ is nonstochastic, or equivalently does not depend on $\omega$, it is sometimes useful to work with a variant formulation of (1.6). With $T=T(\omega)$ for all $\omega$, we obtain

$$
\begin{aligned}
& \text { (1.15) find } x \in R_{+}^{n_{1}}, x \in R^{m_{2}} \text { such that } \\
& A x=b, \quad T x=x \text {, and } \\
& z=c x+\Psi(X) \quad \text { is minimized }
\end{aligned}
$$

where

$$
\Psi(X)=E\{\psi(X, \xi(\omega))\}=\int \psi(X, \xi(\omega)) P(d \omega)
$$

and

$$
\psi(\chi, \xi(\omega))=\inf \left\{q(\omega) y \mid W y=h(\omega)-\chi, y \in R_{+}^{n_{2}}\right\}
$$

This formulation stresses the fact that choosing $x$ corresponds to generating a tender $X=T x$ to be "bid" against the outcomes $h(\omega)$ of random events. The functions $\psi$ and $\Psi$ have basically the same properties as $Q$ and 2 , replacing naturally the set $K_{2}$ by the set $L_{2}=\left\{X=T x \mid x \in K_{2}\right\}=\left\{X \mid \forall h(\omega) \in \Xi_{h}, \exists y \geq 0\right.$ such that $\left.W y=h(\omega)-T x\right\}$. The function $f$ is now given by

$$
f((x, x), \xi)=\left[\begin{array}{ll}
c x+\psi(\chi, \xi) & \text { if } A x=b, T x=\chi, x \geq 0, \\
+\infty & \text { otherwise. }
\end{array}\right.
$$

A significant number of applications have the function $\psi$ separable, i.e. $\psi(X, \xi)=\sum_{i=1}^{m_{2}} \psi_{i}\left(X_{i}, \xi_{i}\right)$ such as in stochastic programs with simple recourse [1, Section 6]. This will substantially simplify the implementation of various approximation schemes described below. When separability is not at hand, it will sometimes be useful to introduce it, by constructing appropriate approximates for $\psi$ or $Q$, see Section 3 . 
Another common feature of stochastic optimization problems, that one should not lose track of when designing approximation schemes, is that the random behavior of the stochastic elements of the problem can often be traced back to a few independent random variables. Typically

$$
\xi(\omega)=\zeta_{1}(\omega) \xi^{1}+\zeta_{2}(\omega) \xi^{2}+\cdots+\zeta_{M}(\omega) \xi^{M}
$$

where the

$$
\left\{\zeta_{i}: \Omega \rightarrow R ; i=1, \ldots, M\right\}
$$

are independent real-valued random variables, and

$$
\xi^{i}=\left(q_{1}^{i}, \ldots, q_{n_{2}}^{i}, h_{1}^{i}, \ldots, h_{m_{2}}^{i}, t_{11}^{i}, \ldots, t_{m_{2} n_{1}}^{i}\right)
$$

are fixed vectors. In fact many applications -- such as those involving scenario analysis -- involve just one such random variable $\zeta(\cdot)$; naturally, this makes the components of the random vector $\xi(\cdot)$ highly dependent. Last, but not least, only rarely do we have in practice adequate statistics to model with sufficient accuracy joint phenomena involving intricate relationships between the components of $\xi$. Hence, we shall devote most of our attention to the independent case, remaining at all times very much aware of the construction (1.19).

This will serve as background to our study of approximation schemes for calculating

$$
E_{f}(x)=\int f(x, \xi(\omega)) P(d \omega)
$$

After taking care of some general convergence results (Section 2), we begin our study with a description of possible approximates of $f$ in the context of stochastic programs with recourse (Section 3.) We then examine the possibility of obtaining lower or upper bounds on $E_{f}$ by means of discretization (of the probability measure) using conditional expectations (Section 4), measures with extremal 
support (Section 5), extremal measures (Section 6) or majorizing probability measures (Section 7). In each case we also sketch out the implementation of the results in the framework of stochastic programs with recourse, relying in some cases on the approximates to $\mathrm{f}$ obtained in Section 3 . In the last section, we give some further error bounds for inf $\mathrm{E}_{\mathrm{f}}$ that require the actual calculation of $\mathrm{E}_{\mathrm{f}}(\mathrm{x})$ at some points. 


\section{CONVERGENCE RESUILTS}

The purpose of this section is to free us at once from any further detailed argumentation involving convergence of solutions, infima, and so on. To do so we rely on the tools provided by epi-convergence. Let $\left\{g ; g^{\nu}, \nu=1, \ldots\right\}$ be a collection of functions defined on $R^{n}$ with values in $\bar{R}=[-\infty,+\infty]$. The sequence $\left\{g^{\nu}, v=1, \ldots\right\}$ is said to epi-converge to $g$ if for all $x \in R^{n}$, we have

$$
\lim \inf g^{\nu}\left(x^{\nu}\right) \geq g(x) \text { for all }\left\{x^{\nu}, \nu=1, \ldots\right\} \text { converging to } x \text {, }
$$
and there exists $\left\{x^{\nu}, \nu=1, \ldots\right\}$ converging to $x$ such that $\lim \sup _{\nu \rightarrow \infty} g^{\nu}\left(x^{\nu}\right) \leq g(x)$.

Note that any one of these conditions implies that $g$, the epi-limit of the $g^{\nu}$, is necessarily lower semicontinuous. The name epi-convergence comes from the fact that the functions $\left\{g^{\nu}, \nu=1, \ldots\right\}$ epi-converge to $g$ if and only if the sets $\left\{\right.$ epi $\left.g^{\nu}, \nu=1, \ldots\right\}$ converge to epi $g=\{(x, \alpha) \mid g(x) \leq \alpha\}$; for more details consult $[2,3]$. Our interest in epi-convergence stems from the following properties [4].

2.3 THEOREM. SUppose a sequence of functions $\left\{\mathrm{g}^{\nu}, \mathrm{v}=1, \ldots\right\}$ epi-converges to $\mathrm{g}$. Then and, if

$$
\lim _{\nu \rightarrow \infty} \sup \left(\inf g^{\nu}\right) \leq \inf g
$$

$$
x^{k} \in \operatorname{argmin} g^{\nu_{k}}=\left\{x \mid g^{\nu} k(x) \leq \inf g^{\nu_{k}}\right\}
$$

for some subsequence of functions $\left\{g^{\nu}, k=1, \ldots\right\}$ and $x=\lim _{k \rightarrow \infty} x^{k}$, it follows that $\mathrm{x} \in \operatorname{argmin} \mathrm{g}$,

and

$$
\lim _{k \rightarrow \infty}\left(\inf g^{{ }^{v} k}\right)=\inf g \text {. }
$$

Moreover, if argmin $g \neq \emptyset$, then $\lim _{\nu \rightarrow \infty}\left(\right.$ inf $g^{\nu}$ ) $=$ inf $g$ if and only if $x \in$ argmin $g$ 
implies the existence of sequences $\left\{\varepsilon_{v} \geq 0, v=1, \ldots\right\}$ and $\left\{x^{v}, v=1, \ldots\right\}$ with

$$
\lim _{v \rightarrow \infty} \varepsilon_{\nu}=0 \text {, and } \quad \lim _{v \rightarrow \infty} x^{\nu}=x \text {, }
$$

such that for all $v=1, \ldots$,

$$
x_{v} \in \varepsilon_{\nu}-\operatorname{argmin} g^{\nu}=\left\{x \mid g^{\nu}(x) \leq \inf g^{\nu}+\varepsilon_{\nu}\right\}
$$

2.5 COROLLARY. Suppose a sequence of functions $\left\{\mathrm{g}^{\nu}, \nu=1, \ldots\right\}$ epi-converges to $g$, and there exists a bounded set $\mathrm{D}$ such that

$$
\operatorname{argmin} g^{\nu} \cap D \neq \emptyset
$$

for alz $\vee$ sufficiently large. Then

$$
\lim _{\nu \rightarrow \infty}\left(\inf g_{\nu}\right)=\inf g
$$

and the minimum of $g$ is attained at some point in the closure of $D$ :

PROOF. Since D is bounded, it follows that there exists a bounded sequence $\left\{x^{v}, v=1, \ldots\right\}$ with

$$
x^{\nu} \epsilon \operatorname{argmin} g^{\nu} \cap D
$$

This means that a subsequence converges $\left\{x^{{ }^{v}}, k=1, \ldots\right\}$ to a point $x$ both in the closure of $D$ and in argmin $g$ as follows from epi-convergence. Theorem 2.3 also yields

$$
\lim _{k \rightarrow \infty} g^{{ }^{v}}\left(x^{v_{k}}\right)=g(x)=\inf g \text {. }
$$

There remains only to argue that the entire sequence $\{($ inf $g \nu), v=1, \ldots\}$ converges to inf $\mathrm{g}$. But this simply follows from the observation that the preceding argument applied to any subsequence yields a further subsequence converging to inf $\mathrm{g}$.

The following proposition provides very useful criteria for verifying epi-convergence. 
2.6 PROPOSITION. [5, Proposition 3.12] Suppose $\left\{\mathrm{g}^{\nu}: \mathrm{R}^{\mathrm{n}} \rightarrow \overline{\mathrm{R}}, \mathrm{v}=1, \ldots\right\}$ is a collection of functions pointwise converging to $g$, i.e. for all $x, g(x)=\lim _{v \rightarrow \infty} g^{\nu}(x)$. Then the $\mathrm{g}^{\nu}$ epi-converge to $\mathrm{g}$, if they are monotone increasing, or monotone decreasing with $g$ lower semicontinuous in this latter case.

For expectation functionals, we obtain the next assertion as a direct consequence of the definition of epi-convergence and Fatou's lemma.

2.7 THEOREM. Suppose $\left\{\mathrm{f} ; \mathrm{f}^{\nu}, \nu=1, \ldots\right\}$ is a collection of functions defined on $\mathrm{R}^{\mathrm{n}} \times \Omega$ with values in $\mathrm{R} \cup\{+\infty\}$ satisfying conditions (1.3) and (1.4), such that for alz $\xi \in \Xi$ the sequence $\left\{\mathrm{f}^{\nu}(\cdot, \xi), v=1, \ldots\right\}$ epi-converges to $\mathrm{f}(\cdot, \xi)$. Suppose moreover that the functions $\mathrm{f}^{\nu}$ are bounded below uniformly. Then the expectation functionals $\mathrm{E}_{\mathrm{f}}$ epi-converge to $\mathrm{E}_{\mathrm{f}}$.

When instead of approximating the functional $f$, we approximate the probability measure $P$, we get the following general result that suits our needs in most applications, see [6, Theorem 3.9], [7, Theorem 3.3].

2.8 THEOREM. Suppose $\left\{\mathrm{P}_{v}, v=1, \ldots\right\}$ is a sequence of probability measures converging in distribution to the probability measure $\mathrm{P}$ defined on $\Omega$, a separable metric space with A the Borel sigma-field. Let

$$
(x, \omega) \mapsto f\left(x, \xi(\omega): \quad R^{n_{x}} \rightarrow R \cup\{+\infty\}\right.
$$

be continuous in $w$ for each fixed $x$ in $k$, where

$$
K=\left\{x \mid E_{f}(x)<+\infty\right\}=\{x \mid f(x, \xi(\omega))<+\infty, a . s .\} \neq \emptyset,
$$

and locally Lipschitz in $\mathrm{x}$ on $\mathrm{K}$ with Lipschitz constant independent of $\omega$. Suppose moreover that for any $\mathrm{x} \in \mathrm{K}$ and $\varepsilon>0$ there exists a compact set $\mathrm{S}_{\varepsilon}$ and $\nu_{\varepsilon}$ such that for all $v \geq v_{\varepsilon}$ 


$$
\int_{\Omega \backslash S_{\varepsilon}}|f(x, \xi(\omega))| P_{\nu}(d \Omega)<\varepsilon,
$$

and with $\mathrm{V}=\{w \mid \mathrm{f}(\mathrm{x}, \mathrm{w})=+\infty\}, \mathrm{P}(\mathrm{V})>0$ if and only if $\mathrm{P}_{\mathrm{V}}(\mathrm{V})>0$. Then the sequence of expectation functionals $\left\{\mathrm{E}_{\mathrm{f}}^{\nu}, v=1, \ldots\right\}$ epi-and pointwise converges to $\mathrm{E}_{\mathrm{f}}$, where

$$
E_{f}^{v}(x)=\int f(x, \xi(\omega)) P_{v}(d \omega)
$$

PROOF. We begin by showing that the $E_{f}^{\nu}$ pointwise converge to $E_{f}$. First let $x \in K$ and set

$$
g(\omega)=f(x, \omega)
$$

From (2.9), it follows that for all $\varepsilon>0$, there is a compact set $S_{\varepsilon}$ and index $\nu_{\varepsilon}$ such that for a11 $\nu \geq \nu_{\varepsilon}$

$$
\int_{\Omega \backslash S_{\varepsilon}}|g(\omega)| P_{\nu}(d \omega)<\varepsilon .
$$

Let $M_{\varepsilon}=\sup _{\omega \in S_{\varepsilon}}|g(\omega)|$. We know that $M_{\varepsilon}$ is finite since $S_{\varepsilon}$ is compact and $g$ is continuous, recal1 that $x \in K$. Let $g^{\varepsilon}$ be a truncation of $g$ defined by

$$
g^{\varepsilon}(\omega)=\left\{\begin{array}{lll}
g(\omega) & \text { if } & |g(\omega)| \leq M_{\varepsilon} \\
M_{\varepsilon} & \text { if } & g(\omega)>M_{\varepsilon} \\
-M_{\varepsilon} & \text { if } & g(\omega)<-M_{\varepsilon}
\end{array}\right.
$$

The function $g^{\varepsilon}$ is bounded and continuous and we have that for all $\omega \in \Omega$

$$
\left|g^{\varepsilon}(\omega)\right| \leq|g(\omega)|
$$

Hence from the convergence in distribution of the $\mathrm{P}_{\nu}$

$$
\lim _{v \rightarrow \infty}\left[\beta_{\nu}^{\varepsilon}=\int_{\Omega} g^{\varepsilon}(\omega) P_{\nu}(d \omega)\right]=\int_{\Omega} g^{\varepsilon}(\omega) P(d \omega)=\beta^{\varepsilon}
$$

and also for al1 $\nu \geq \nu_{\varepsilon}$, 
Now let

$$
\int_{\Omega \backslash S_{\varepsilon}} g^{\varepsilon}(\omega) P_{v}(d \omega)<\varepsilon
$$

$$
\beta_{v}=E_{f}^{\nu}(x)=\int_{S_{\varepsilon}} g(\omega) P_{v}(d \omega)+\int_{\Omega \backslash S_{\varepsilon}} g(\omega) P_{V}(d \omega) .
$$

We have that for all $\nu \geq \nu_{\varepsilon}$

$$
\left|\beta_{\nu}-\beta_{\nu}^{\varepsilon}\right|=\left|\int_{\Omega \backslash S_{\varepsilon}}\left[g(\omega)-g^{\varepsilon}(\omega)\right] P_{\nu}(d \omega)\right|<2 \varepsilon
$$

and also that

$$
\left|E_{f}(x)-\beta^{\varepsilon}\right|<2 \varepsilon
$$

Combining the two last inequalities with (2.10) shows that for all $\varepsilon>0$, there exists $\nu_{\varepsilon}$ such that for all $\nu \geq \nu_{\varepsilon}$

$$
\left|E_{f}(x)-\beta_{v}\right|<6 \varepsilon,
$$

and thus for a11 $x \in K$,

$$
\lim _{\nu \rightarrow \infty} E_{f}^{\nu}(x)=E_{f}(x) .
$$

If $x \notin K$, this means that

$$
P[V=\{\omega \mid f(x, \xi(\omega))=+\infty\}]>0
$$

which also means that for all $v$

$$
\mathrm{P}_{V}(\mathrm{~V})>0,
$$

from which it follows that for all $v$

$$
\lim _{\nu \rightarrow+\infty} E_{f}^{\nu}(x)=+\infty=E_{f}(x)
$$

And thus, for all $x \in R^{n}, E_{f}(x)=\lim _{v \rightarrow \infty} E_{f}^{v}(x)$. This gives us not only pointwise convergence, but also condition (2.2) for epi-convergence. 
To complete the proof, it thus suffices to show that condition (2.1) is satisfied for all $x \in K$. The function $x \mapsto f(x, \xi(\omega))$ is Lipschitzian on $K$, with Lipschitz constant $L$ independent of $\omega$. For any pair $x, x^{\nu}$ in $K$, we have that for all $\omega$

$$
\left|f(x, \xi(\omega))-f\left(x^{\nu}, \xi(\omega)\right)\right| \leq L \operatorname{dist}\left(x, x^{\nu}\right)
$$

which implies that

$$
f(x, \xi(\omega))-L \operatorname{dist}\left(x, x^{\nu}\right) \leq f\left(x^{\nu}, \xi(\omega)\right)
$$

Let us now take $x^{\nu}$ as part of a sequence $\left\{x^{\nu}, \nu=1, \ldots\right\}$ converging to $x$. Integrating on both sides of the preceding inequality and taking $\lim _{\nu \rightarrow \infty}$ inf, we get

$$
\begin{aligned}
E_{f}(x) & =\lim _{\nu \rightarrow \infty} E_{f}^{\nu}(x)-L \lim _{\nu \rightarrow \infty} \operatorname{dist}\left(x, x^{\nu}\right) \\
& =\underset{\nu \rightarrow \infty}{\lim \inf }\left(E_{f}^{\nu}(x)-L \operatorname{dist}\left(x, x^{\nu}\right)\right) \\
& \leq \underset{\nu \rightarrow \infty}{\lim \inf } E_{f}^{\nu}\left(x^{\nu}\right),
\end{aligned}
$$

which completes the proof. $\square$

2.11 APPLICATION. Suppose $\left\{\mathrm{P}_{v}, v=1, \ldots\right\}$ is a sequence of probability measures that converge in distribution to $\mathrm{P}$, all with compact support $\Omega$. Suppose

$$
Q^{\nu}(x)=\int Q(x, \xi(\omega)) P_{v}(d \omega)
$$

with the recourse function $Q$ defined by (1.8) and $Q$ by (1.7). Then the $Q^{\nu}$ both epi- and pointwise converge to 2 .

It suffices to observe that the conditions of Theorem 2.8 are satisfied. The continuity of $Q(x, \xi)$ with respect to $\xi$ (for $x \in K_{2}$ ) follows from (1.11) and (1.12). The Lipschitz property with respect to $\mathrm{x}$ is obtained from [1, Theorem 7.7]; the proof of that Theorem also shows that the Lipschitz constant is independent of $\xi$, consult also [8]. 
2.12 IMPLEMENTATION. From the preceding results it follows that we have been given great latitude in the choice of the probability measures that approximate $P$. However, in what follows we concern ourselves almost exclusively with discrete probability measures. The basic reason for this is that the form of $f(x, \xi)$-or $Q(x, \xi)$ in the context of stochastic programs with recourse -- renders the numerical evaluation of $E_{f}$ (or $E_{f}^{V}$ ) possible only if the integral is actually a (finite) sum. Only in highly structured problems, such as for stochastic programs with simple recourse [9], may it be possible and profitable to use other approximating measures . 


\section{APPROXIMATING THE RECOURSE FUNCTION Q}

When $f$ is convex in $\xi$, it is possible to exploit this property to obtain simple but very useful lower bounding approximates for $\mathrm{E}_{\mathrm{f}}$.

3.1 PROPOSITION. Suppose $\xi \leftrightarrow f(x, \xi)$ is convex, $\left\{\xi^{\ell}, \ell=1, \ldots, v\right\}$ is a finite collection of points in $\Xi$, and for $\ell=1, \ldots$,

$$
v^{\ell} \in \partial_{\xi} f\left(x, \xi^{l}\right)
$$

i.e. $v^{\ell}$ is a subgradient of $f(x, \cdot)$ at $\xi^{\ell}$. Then

$$
\mathrm{E}_{\mathrm{f}}(\mathrm{x}) \geq \mathrm{E}\left\{\max _{1 \leq \ell \leq \nu}\left[\mathrm{v}^{\ell} \xi(\omega)+\left(\mathrm{f}\left(\mathrm{x}, \xi^{\ell}\right)-\mathrm{v}^{\ell} \xi^{\ell}\right)\right]\right\} .
$$

PROOF. To say that $v^{l}$ is a subgradient of the convex function of $f(x, \cdot)$ at $\xi^{l}$, means that

$$
f(x, \xi)-f\left(x, \xi^{l}\right) \geq v^{l}\left(\xi-\xi^{l}\right) .
$$

Since this holds for every $l$, we obtain

$$
f(x, \xi) \geq \max _{1 \leq \ell \leq \nu}\left[v^{l} \xi+\left(f\left(x, \xi^{l}\right)-v^{l} \xi^{l}\right)\right] .
$$

Integrating on both sides yields (3.2).

3.3 APPLICATION. Consider the stochastic progrom with recourse (1.6) and suppose that only $h$ and $T$ are stochastic. Let $\left\{\xi^{l}=\left(h^{l}, T^{l}\right), l=1, \ldots, v\right\}$ be a finite number of realizations of $h$ and $T, x \in K_{2}$ and for $l=1, \ldots, v$,

$$
\pi^{\ell} \in \operatorname{argmax}\left[\pi\left(h^{\ell}-T^{l} x\right) \mid \pi W \leq q\right] .
$$

Then

(3.4) $\quad 2(x) \geq E\left\{\max _{1 \leq \ell \leq \nu} \pi^{\ell}(h(\omega)-T(\omega) x)\right\}$. 
This is a direct corollary of Proposition 3.1. We give an alternative proof which could be of some help in the design of the implementation. Since $x \in K_{2}$, for every $\xi=(h, T)$ in $\Xi$, the linear program

$$
\begin{aligned}
& \text { find } \pi \in R^{m_{2}} \text { such that } \pi W \leq q \\
& \text { and } w=\pi(h-T x) \text { is maximized }
\end{aligned}
$$

is bounded, given naturally that it is feasible as follows from assumption (1.10). Hence for $\ell=1, \ldots, \nu$,

$$
Q\left(x, \xi^{\ell}\right)=\pi^{\ell}\left(h^{\ell}-T^{\ell} x\right)
$$

and moreover since $\pi^{l}$ is a feasible solution of the linear program (3.5), for all $\xi \in \Xi$

$$
Q(x, \xi) \geq \pi^{\ell}(h-T x) .
$$

Since this holds for every $l$, we get

$$
Q(x, \xi) \geq \max _{1 \leq \ell \leq \nu^{\prime}} \pi^{\ell}(h-T x)
$$

Integrating on both sides yields $(3.4)$.

3.6 IMPLEMENTATION. In general finding in expression (3.4), the maximum for each $\xi$-- or equivalently for each $(h, T) \epsilon \Xi--$ could be much too involved. But we may assign to each $\pi^{\ell}$ a subregion of $\Xi$, without resorting to (exact) maximization. The lower bound may then not be as tight as (3.4), but we can refine it by taking successively finer and finer partitions. However, one should not forget that (3.4) involves a rather simple integral and the expression to the right could be evaluated numerically to an acceptable degree of accuracy, without major difficulties. Note that the calculation of this lower bound imposes no limitations on the choice of the $\xi^{\ell}$. However, it is obvious that a well-chosen spread of the $\left\{\xi^{\ell}, \ell=1, \ldots, \nu\right\}$ will yield a better approximation. For example, the $\xi^{\ell}$ could be 
the conditional expectation of $\xi(\cdot)$ with respect to a partitioning $S=\left\{S_{\ell}, \ell=1, \ldots, \nu\right\}$ of $\Xi$ which assigns to each $\mathrm{S}_{\ell}$ about the same probability. Also the use of a larger collection of points, i.e. increasing $\nu$, will also yield a better lower bound.

3.7 CONVERGENCE. Suppose that $\xi \leftrightarrow f(x, \xi)$ is convex. For each $v=1, \ldots$, let $S^{\nu}=\left\{S_{\ell}^{\nu}, \ell=1, \ldots, L_{\nu}\right\}$ denote a partition of $\Xi$ with

$$
\xi^{\nu \ell}=E\left\{\xi(\omega) \mid S_{\ell}^{\nu}\right\}
$$

the conditional expectation of $\xi(\cdot)$ given $S_{\ell}^{\nu}$. Suppose moreover that $S^{\nu} \subset S^{\nu+1}$ and that

(3.8) $\lim _{\nu \rightarrow \infty}\left(\max _{1 \leq \ell \leq L_{\nu}} P\left[\omega \mid \xi(\omega) \in S_{\ell}^{\nu}\right]\right)=0$.

Then, with $v^{\nu \ell} \in \partial_{\xi} f\left(x, \xi^{\nu l}\right)$ and

$$
E_{f}^{\nu}(x)=E\left\{\max _{1 \leq \ell \leq L_{\nu}}\left[v^{\ell \nu} \xi(\omega)+f\left(x, \xi^{\nu \ell}\right)-v^{\nu \ell} \xi^{\nu \ell}\right]\right\},
$$

we have that the sequence of functions $\left\{\mathrm{E}_{f}^{\nu}, v=1, \ldots\right\}$ is monotone increasing, and for alz x:

$$
E_{f}(x)=\lim _{\nu \rightarrow \infty} E_{f}^{v}(x)
$$

Hence the sequence $\left\{\mathrm{E}_{\mathrm{f}}^{\nu}, \nu=1, \ldots\right\}$ is both pointwise- and epi-convergent.

PROOF. From Proposition 3.1, it follows that $E_{f}^{\nu} \leq E_{f}$ for all $v$. The inequality

$$
E_{f}^{\nu} \leq E_{f}^{\nu+1} \leq E_{f}
$$

then follows simply from the fact that $S^{\nu+1} \supset S^{\nu}$. Now observe that

$$
\max _{1 \leq \ell \leq L_{v}}\left[v^{\nu \ell} \xi+f\left(x, \xi^{\nu \ell}\right)-v^{\nu \ell} \xi^{\nu \ell}\right] \geq g^{\nu}(x, \xi)
$$

where $\mathrm{g}^{\nu}$ is defined as follows:

$$
g^{\nu}(x, \xi)=v^{\nu \ell} \xi+f\left(x, \xi^{\nu \ell}\right)-v^{\nu l} \xi^{\nu \ell} \text { if } \xi \in S_{\ell}^{\nu} \text {. }
$$


It follows that

$$
E_{f}^{\nu}(x) \geq E\left\{g^{\nu}(x, \xi(\omega))\right\}=\sum_{\ell=1}^{L_{\nu}} P\left[\xi(\omega) \in S_{l}^{\nu}\right] f\left(x, \xi^{\nu \ell}\right)
$$

which gives us

$$
E_{f}(x) \geq \lim _{\nu \rightarrow \infty} E_{f}^{\nu}(x) \geq \lim _{\nu \rightarrow \infty} E\left\{g^{\nu}(x, \xi(\omega))\right\}=E_{f}(x)
$$

the last equality following from assumption (3.8).

We have thus shown that the sequence $\left\{E_{f}^{\nu}, v=1, \ldots\right\}$ is monotone increasing and pointwise converges, and this implies epi-convergence, see Proposition 2.6.

If $\mathrm{f}(\mathrm{x}, \cdot)$ is concave, the inequality in (3.2) is reversed and, instead of a lower bound on $E_{f}$, we obtain an upper bound. In fact, we can again use Proposition 3.1 , but this time applied to $-f$.

3.11 APPLICATION. Consider the stochastic progrom with recourse (1.6) and suppose that only the vector $\mathrm{q}$ is stochastic. Let $\left\{\xi^{\ell}=\mathrm{q}^{\ell}, \ell=1, \ldots, v\right\}$ be a finite number of reatizations of $q, x \in K_{2}$ and for $l=1, \ldots, v$

$$
y^{\ell} \in \operatorname{argmin}\left[q^{\ell} y \mid W y=p-T x, y \geq 0\right] .
$$

Then

$$
Q(x) \leq E\left\{\min _{1 \leq \ell \leq \nu} q(\omega) y^{\ell}\right\}
$$

Again this is really a corollary of Proposition 3.1. A slightly different proof proceeds as follows: Note that for all $q=\xi \in \Xi$, for every $\ell, y^{\ell}$ is a feasible, but not necessarily optimal, solution of the linear program

find $y \in R_{+}^{n_{2}}$ such that $W y=p-T x$, and $w=q y$ is minimized.

Hence

$$
Q(x, \xi) \leq \min _{1 \leq \ell \leq \nu} q y^{\ell}
$$

from which (3.12) follows by integrating on both sides. 
The remarks made about Implementation 3.6 and the arguments used in Convergence 3.7 still apply to the concave case since we are in the same setting as before provided we work with $-f$ or $-Q$.

Proposition 3.1 provides us with a lower bound for $E_{f}$ when $\xi \mapsto f(x, \xi)$ is convex, the next result yields an upper bound.

3.13 PROPOSITION. Suppose $\xi \leftrightarrow f(x, \xi)$ is convex, $\left\{\xi^{\ell}, l=1, \ldots, \nu\right\}$ is a finite collection of points in $\Xi$. Then

$$
E_{f}(x) \leq E_{f}^{\nu}(x)=\int f^{\nu}(x, \xi(\omega)) P(d \omega)
$$

where

$$
f^{\nu}(x, \xi)=\inf _{\lambda \in R_{+}^{\nu}}\left[\sum_{\ell=1}^{\nu} \lambda_{\ell} f\left(x, \xi^{\ell}\right) \mid \xi=\sum_{\ell=1}^{\nu} \lambda_{\ell} \xi^{\ell}, 1=\sum_{\ell=1}^{\nu} \lambda_{\ell}\right] .
$$

If the function $\xi \mapsto f(x, \xi)$ is sublinear, the $f^{\nu}$ can be defined as folzows:

$$
f^{\nu}(x, \xi)=\inf _{\lambda \in R_{+}^{\nu}}\left[\sum_{\ell=1}^{\nu} \lambda_{\ell} f\left(x, \xi^{l}\right) \mid \xi=\sum_{\ell=1}^{\nu} \lambda_{\ell} \xi^{\ell}\right] \text {. }
$$

(Note that $f^{\nu}(x, \xi)$ is $+\infty$ if the corresponding program is infeasible.)

PROOF. Convexity implies that for a11 $\lambda_{1} \geq 0, \ldots, \lambda_{V} \geq 0$ with $\sum_{\ell=1}^{V} \lambda_{\ell}=1$, and $\xi=\sum_{\ell=1}^{\nu} \lambda_{\ell} \xi^{\ell}$ we have

(3.17) $\quad f(x, \xi) \leq \sum_{\ell=1}^{\nu} \lambda_{\ell} f\left(x, \xi^{\ell}\right)$

from which (3.14) follows using (3.15). Sublinearity (convexity and positive homogeneity) also yields (3.17) but this time without $\sum_{\ell=1}^{V} \lambda_{\ell}=1$, and this in turn yields (3.14) using (3.16) this time.

3.18 APPLICATION. Ray functions. Consider the stochastic program with recourse in the form (1.15) and suppose that only $h$ is stochastic, i.e. with fixed matrix $T$ and recourse cost coefficients $q$. Now suppose that for given $\chi$, we have the values of $\left\{\psi\left(\chi, \xi^{\ell}=h^{\ell}\right), \ell=1, \ldots, \nu\right\}$ for a finite collection of realizations of $h(\cdot)$. 
Let $\xi \in \Xi$ and define

$$
\psi^{\nu}(x, \xi)=\inf { }_{\lambda \in R_{+}^{\nu}}\left[\sum_{\ell=1}^{\nu} \lambda_{\ell} \psi\left(x, \xi^{\ell}\right) \mid \xi=x+\sum_{\ell=1}^{\nu} \lambda_{\ell}\left(\xi^{\ell}-x\right)\right] .
$$

Then

$$
\Psi(x) \leq \Psi^{\nu}(x)=\int \psi^{\nu}(x, \xi(\omega)) P(d \omega)
$$

The above follows from the second part of Proposition 3.13 provided we observe that from the definition (1.17) of $\psi$, we have that

$$
h \mapsto \psi(\chi, h+\chi)
$$

is sublinear. From this it follows that for any $\lambda \in R_{+}^{\nu}$

$$
\psi(x,(\xi-x)+x) \leq \sum_{\ell=1}^{v} \lambda_{\ell} \psi\left(x,\left(\xi^{\ell}-x\right)+x\right)
$$

whenever

$$
\xi-x=\sum_{\ell=1}^{\nu} \lambda_{\ell}\left(\xi^{\ell}-\chi\right)
$$

and this leads to the construction of $\psi^{\nu}$ in (3.19).

3.20 IMPLEMENTATION. Finding for each $\xi$, the optimal value of a linear program as required by the definition of $\psi^{\nu}$ in (3.19), could involve much more work than is appropriate to invest in the computation of an upper bound. One way to remedy this is to subdivide $\Xi$ such that each $\xi$ is automatically assigned to a particular region spanned by a subset of the $\left\{\xi^{\ell}, \ell=1, \ldots, \nu\right\}$ or to the subset whose points are such that

$$
\xi-x \notin \operatorname{pos}\left(\xi^{1}-x, \ldots, \xi^{\nu}-\chi\right)=\left\{t \mid t=\sum_{\ell=1}^{v} \lambda_{\ell}\left(\xi^{\ell}-x\right), \lambda \in R_{+}^{\nu}\right\} .
$$

One case in which all of this falls nicely into place is when a stochastic program with recourse of type (1.15) can be approximated by a stochastic program with simple recourse [1, Section 6] where the function $\psi(\chi, \xi)$ is separable, 
(3.21) $\quad \psi(x, \xi)=\sum_{i=1}^{m_{2}} \psi_{i}\left(x_{i}, \xi_{i}\right)$

and

(3.22) $\psi_{i}\left(x_{i}, \xi_{i}\right)=\inf \left[q_{i}^{+} y_{i}^{+}+q_{i}^{-} y_{i}^{-} \mid y_{i}^{+}-y_{i}^{-}=h_{i}-x_{i}, y_{i}^{+} \geq 0, y_{i}^{-} \geq 0\right]$,

here $\xi_{i}=\left(q_{i}^{+}, q_{i}^{-}, h_{i}\right)$. The function $\Psi$ is then also separable and can be expressed as

$$
\Psi(X)=\sum_{i=1}^{m_{2}} \Psi_{i}\left(X_{i}\right),
$$

where

$$
\Psi_{i}\left(\chi_{i}\right)=E\left\{\psi_{i}\left(X_{i}, \xi_{i}(\omega)\right)\right\}
$$

(This is the linear version of the simple recourse problem.)

3.23 APPLICATION. Approximation by simple recourse. Consider a stochastic program with recourse of the type (1.15), with only $h$ stochastic and complete recourse $[1$, Section 6], this means that the recourse matrix $W$ is such that

$$
\operatorname{pos} W=\{t \mid t=W y, y \geq 0\}=R^{m_{2}},
$$

i.e. the recourse problem is feasible whatever be $h$ or $\chi$. For $i=1, \ldots, m_{2}$, define (3.24) $\quad q_{i}^{+}=\inf \left\{q y \mid\right.$ wy $\left.=e^{i}, y \geq 0\right\}$,

and

(3.25) $\quad q_{i}^{-}=\inf \left\{q y \mid w=-e^{i}, y \geq 0\right\}$,

where $e^{i}$ is the unit vector with a 1 in the $i^{\text {th }}$ position, i.e.

$$
e^{i}=(0, \ldots, 0,1,0, \ldots, 0)^{\mathrm{T}} \text {. }
$$

The recourse function $\psi(\chi, \xi)$ is then approximated by the recourse function (3.21) of a stochastic program with simple recourse using for $q_{i}^{+}$and $q_{i}^{-}$the values defined by (3.24) and (3.25). This is a special case of the ray functions built in Application 3.18; each $(\xi-\chi)$ falls in a given orthant and is thus automatically assigned a particular positive linear combination of the chosen 
points $\left( \pm e^{i}-x, i=1, \ldots, m_{2}\right)$. To improve the approximation we have to introduce additional vectors $\xi^{\ell}$, which brings us back to the more general situation described in Application 3.18 .

3.26 APPLICATION. Consider a stochastic program with recourse of type (1.15), with only q stochastic. The function

$$
\mathrm{q} \mapsto \psi(x, \mathrm{q}=\xi): \mathrm{R}^{\mathrm{n} 2} \rightarrow \overline{\mathrm{R}}
$$

is not only concave and polyhedral (1.12), it is also positively homogeneous. For any finite collection $\left\{\xi^{\ell}=q^{\ell}, \ell=1, \ldots, \nu\right\}$ we have that

$$
\psi(x, q) \geq \sup _{\lambda \in R_{+}^{v}}\left[\sum_{\ell=1}^{v} \lambda_{\ell} \psi\left(x, q^{\ell}\right) \mid q=\sum_{\ell=1}^{v} \lambda_{\ell} q^{\ell}\right] .
$$

This again follows directly from Proposition 3.13 ; note that $\psi\left(x, q^{l}\right)=q^{l} y^{l}$ where $y^{\ell} \in \operatorname{argmin}\left[q^{\ell} y \mid w y=h-x, y \geq 0\right]$.

3.28 IMPLEMENTATION. Calculating for each $q$, the upper bound provided by (3.27) may be prohibitive. We could assign each $q \in \Xi$ to some subregion of $\Xi$ spanned by the positive combinations of some of the $\left\{q^{\ell}, \ell=1, \ldots, v\right\}$. Such a bound is much easier to obtain but clearly not as sharp as that generated by (3.27).

Another approach to getting upper and lower bounds for stochastic programs with recourse is to rely on the pairs programs as introduced in [10, Section 4]. One relies again on convexity properties and once again one needs to distinguish between $(h, T)$ stochastic, and $q$ stochastic. To begin with, let us consider $h$, and T stochastic. For every $(h, T)=\xi \in \Xi$, and $(\hat{h}, \hat{T})=\hat{\xi} \in$ co $\Xi$ (the convex hull of $\Xi$ ), let

$$
\begin{aligned}
& p(\hat{\xi}, \xi)=\quad \text { inf }\left[c x+\hat{p} q \hat{y}+(1-\hat{p}) q y_{\xi}\right] \\
& \text { such that } A x \\
& =\mathrm{b} \\
& \hat{\mathrm{T} x}+w \hat{y} \quad=\hat{h} \\
& \mathrm{Tx} \quad+\mathrm{Wy}_{\xi}=\mathrm{h} \\
& x \geq 0, \hat{y} \geq 0, y_{\xi} \geq 0 \text {, }
\end{aligned}
$$


with $\hat{p} \in[0,1]$. If (1.6) is solvable, so is (3.29) as follows from [1, Theorem 4.6]. Suppose $x^{0}$ solves $(1.6)$ and for a $11 \xi=(h, T)$, let

$$
y^{o}(\xi) \in \operatorname{argmin}_{y \in \mathrm{R}_{+}} \mathrm{n}_{2}[q y \mid W y=h-T x] .
$$

It is well-known that $\mathrm{y}^{\mathrm{o}}(\xi)$ can be chosen so that $\xi \leftrightarrow \mathrm{y}^{\mathrm{o}}(\xi)$ is measurable

$[1$, Section 3]. Now suppose

$$
\bar{\xi}=(\overline{\mathrm{h}}, \overline{\mathrm{T}})=\mathrm{E}\{\xi\}
$$

and

$$
\bar{y}=E\left\{y^{0}(\xi)\right\}
$$

The triple $\left(x^{0}, \bar{y}, y^{o}(\xi)\right)$ is a feasible, but not necessarily optimal, solution of the linear program (3.29) when $(\hat{h}, \hat{T})=(\bar{h}, \bar{T})$. Hence

$$
\rho(\bar{\xi}, \xi) \leq c x^{\circ}+\hat{p} q \vec{y}+(1-\hat{p}) q y^{\circ}(\xi)
$$

and integrating on both sides, we obtain

$$
\mathrm{E}\{\rho(\bar{\xi}, \xi)\} \leq \mathrm{cx}{ }^{o}+2\left(\mathrm{x}^{\mathrm{o}}\right)
$$

This bound can be refined in many ways: first, instead of just using one point $\bar{\xi}$, one could use a collection of points obtained as conditional expectations of a partition of $\Xi$, and create a pairs program for each subregion of $\Xi$. Second, instead of just one additional point $\hat{\xi}$, we could use a whole collection $\left\{\hat{\xi}^{1}, \ldots, \hat{\xi}^{\vee}\right\}$ to build a program of the type $(3.29)$. All of this is described in detail in [10] for the case when only $h$ is stochastic but can easily be generalized to the case $h$ and $T$ stochastic.

When only $q$ is stochastic, we consider a dual version of (3.29), viz. for every $q=\xi \in \Xi$ and $\hat{q}=\hat{\xi} \in$ co $\Xi$, let

$$
\begin{aligned}
\text { (3.31) } \rho^{\mathrm{d}}(\hat{\xi}, \xi)=\sup _{\text {such that } \sigma \mathrm{A}+\hat{\pi} \mathrm{T}}\left[\sigma \mathrm{b}+\hat{\mathrm{p}} \hat{\pi} \mathrm{h}+(1-\hat{\mathrm{p}}) \pi \xi^{\mathrm{h}}\right] & \leq \mathrm{c} \\
\hat{\pi} W & \leq \hat{\mathrm{q}} \\
& { }^{\pi} \xi^{W} \leq \mathrm{q}
\end{aligned}
$$


with $\hat{\mathrm{p}} \in[0,1]$. The same arguments as above with $\hat{\xi}=\bar{\xi}$, but relying this time on the dual [11] of problem (1.6), lead to

(3.32) $E\left\{\rho^{\mathrm{d}}(\bar{\xi}, \xi)\right\} \geq c x^{\mathrm{o}}+2\left(\mathrm{x}^{\mathrm{o}}\right):=\inf (\mathrm{c} \cdot+2)$. 
4. DISCRETIZATION OF THE PROBABILITY MEASURE P THROUGH CONDITIONAL EXPECTATIONS

Jensen's inequality for convex functions is the basic tool to obtain lower bounds for $E_{f}$ when $f(x, \cdot)$ is convex or upper bounds when $E_{f}$ is concave. Here, it leads to the use of (molecular) probability measures concentrated at conditional expectation points. In the context of stochastic programming this was first done by Madansky [12] and further refined by Kall [13] and Huang, Vertinsky and Ziemba [14].

4.1 PROPOSITION. Let $S=\left\{S^{\ell}, l=1, \ldots, v\right\}$ be a partition of $\Xi$, with

$$
\xi^{\ell}=\mathrm{E}\left\{\xi(\omega) \mid \mathrm{S}^{\ell}\right\} \quad \text { and } \quad \mathrm{p}_{\ell}=\mathrm{P}\left[\xi(\omega) \in \mathrm{S}^{\ell}\right] \text {. }
$$

Suppose first that $\xi \mapsto f(x, \xi)$ is convex. Then

$$
E_{f}(x) \geq \sum_{\ell=1}^{\nu} p_{\ell} f\left(x, \xi^{\ell}\right)
$$

If $\xi \mapsto f(x, \xi)$ is concave, then

$$
E_{f}(x) \leq \sum_{\ell=1}^{v} p_{\ell} f\left(x, \xi^{\ell}\right)
$$

PROOF. Follows from the iterated application of Jensen's inequality: $f(x, E\{\xi(\omega)\}) \leq E\{f(x, \xi(\omega))\}$ when $f(x, \cdot)$ is convex; consult [15] .

4.4 APPLICATION. Consider the stochastic program with recourse with only $h$ and $T$ stochastic. With $S=\left\{S^{\ell}, \ell=1, \ldots, \nu\right\}$ a partition of $\Xi$ and for $\ell=1, \ldots, \nu, 1$ et

$$
\xi^{\ell}=\left(h^{\ell}, T^{\ell}\right)=E\left\{(h(\omega), T(\omega)) \mid S^{\ell}\right\}
$$

and $\mathrm{P}_{\ell}=\mathrm{P}\left[\xi(\omega) \in \mathrm{S}^{\ell}\right]$. As follows from (1.11) and (4.2), we obtain

$$
\sum_{\ell=1}^{v} p_{\ell} Q\left(x, \xi^{\ell}\right) \leq 2(x)
$$

and thus if

$$
\left.z^{\nu}=\inf f_{x \in R^{n}} n_{l=1}^{\nu} p_{\ell} Q\left(x, \xi^{\ell}\right) \mid A x=b, x \geq 0\right]
$$


where

$$
Q\left(x, \xi^{l}\right)=\inf _{y \in R^{2}} n_{2}\left[q y \mid W y=h^{l}-T^{l} x, y \geq 0\right],
$$

we have that

$$
z^{\nu} \leq z^{*}=\inf [c x+2(x) \mid A x=b, x \geq 0] .
$$

Each $z^{\nu}$ is thus a lower bound for the optimal value of the stochastic program. (An alternative derivation of (4.5) relying on the dual of the recourse problem that defines $Q(x, \xi)$ appears in $[16]$.

4.6 CONVERGENCE. Suppose $S^{\nu}=\left\{S^{\ell}, \ell=1, \ldots, \nu\right\}$ for $\nu=1, \ldots$, are partitions of $\Xi$ with $S^{\nu} \subset S^{\nu+1}$ and chosen so that the $P_{\nu}, \nu=1, \ldots$ converge in distribution to $P$. The $P_{V}$ are the (molecular) probability distributions that assign probability $\mathrm{p}_{\ell}=\mathrm{P}\left[\xi(\omega) \in \mathrm{S}^{\ell}\right]$ to the event $\left[\xi(\omega)=\xi^{\ell}\right]$ where $\xi^{\ell}$ is the conditional expectation (with respect to P) of $\xi(\bullet)$ given that $\xi(\omega) \in \mathrm{S}^{\ell}$. The epi-convergence of the $\left\{2^{\nu}, v=1, \ldots\right\}$ to 2 , with the accompanying convergence of the solutions, follows from Theorem 2.8, where

$$
Q^{\nu}(x)=\sum_{\ell=1}^{\nu} P_{\ell} Q\left(x, \xi^{\ell}\right)=\int Q(x, \xi(\omega)) P_{\nu}(d \omega)
$$

To make use of these results we need to develop a sequential partitioning scheme for $\Xi$, i.e. given a partition $S^{\nu}$ of $\Xi$ how should it be refined so as to improve the approximation to 2 as much as possible. P. Kall has also worked out various refinement schemes [17].

4.7 IMPLEMENTATION. Stochastic programs with simple recourse, with h stochastic, $\mathrm{q}$ and $\mathrm{T}$ are fixed. Recall that for a stochastic program with simple recourse $\Psi$ takes on the form:

$$
\Psi(x)=E\left\{\sum_{i=1}^{m_{2}} \psi_{i}\left(x_{i}, \xi_{i}\right)\right\},
$$


where $\xi_{i}=h_{i}$ and, as follows from $(3.21)$,

$$
\psi_{i}\left(x_{i}, \xi_{i}\right)=\left[\begin{array}{cl}
q_{i}^{+}\left(h_{i}-x_{i}\right) & \text { if } h_{i} \geq x_{i}, \\
q_{i}^{-}\left(x_{i}-h_{i}\right) & \text { if } h_{i} \leq x_{i} \cdot
\end{array}\right.
$$

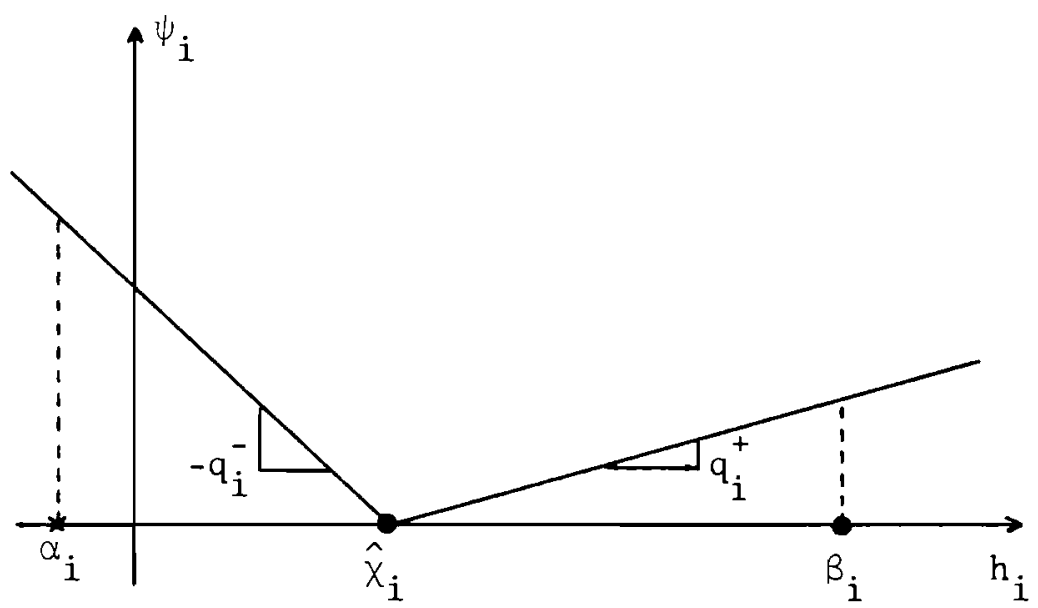

4.8. Figure: The function $\psi_{i}\left(x_{j}, \cdot\right)$

Let $\left[\alpha_{i}, \beta_{i}\right]$ be the support of the realizations of $h_{i}(\cdot)$, possibly an unbounded interval. If we are only interested in a lower bound for $\Psi$ that approximates it as closely as possible at the point $\hat{x}$, then the optimal partitioning of $\left[\alpha_{i}, \beta_{i}\right]$ is given by

$$
s_{i}^{1}=\left[\alpha_{i}, \hat{x}_{i}\right] \text { and } s_{i}^{2}=\left[\hat{x}_{i}, \beta_{i}\right] \text {. }
$$

In this way the approximating function $\Psi_{i}^{a}$ takes on the form:

$$
\psi_{i}^{a}\left(x_{i}\right)= \begin{cases}q_{i}^{+} \bar{h}{ }_{i}-q_{i}^{+} x_{i}, & \text { if } x_{i} \leq h_{i}^{1} \\ \left(q_{i}^{+} h_{i}^{2} p_{i 2}-q_{i}^{-} h_{i}^{1} p_{i 1}\right)+\left(q_{i}^{-} p_{i 1}-q_{i}^{+} p_{i 2}\right) x_{i}, & \text { if } h_{i}^{1} \leq x_{i} \leq h_{i}^{2} \\ -q_{i}^{-} \overline{h_{i}}+q_{i}^{-} x_{i}, & \text { if } x_{i} \geq h_{i}^{2}\end{cases}
$$

where for $\ell=1,2$,

$$
h_{i}^{\ell}=E\left\{h_{i}(\omega) \mid S^{\ell}\right\} \quad \text { and } \quad p_{i \ell}=P\left[h_{i}(\omega) \in S^{\ell}\right] \text {, }
$$

and $\bar{h}_{i}=E\left\{h_{i}(\omega)\right\}$. Note that 


$$
\begin{aligned}
\Psi_{i}\left(\hat{x}_{i}\right) & =q_{i}^{-} \int_{h_{i}(\omega)<\hat{x}_{i}}\left(\hat{x}_{i}-h_{i}(\omega)\right) P(d \omega)+q_{i}^{+} \int_{h_{i}(\omega) \geq \hat{x}_{i}}\left(h_{i}(\omega)-\hat{x}_{i}\right) P(d \omega) \\
& =\Psi_{i}^{a}\left(\hat{x}_{i}\right) .
\end{aligned}
$$

Thus $\Psi_{i}^{a} \leq \Psi_{i}$ with equality holding for $x_{i} \leq \alpha_{i}, x_{i} \geq \beta_{i}$ and at $x_{i}=\hat{x}_{i}$.

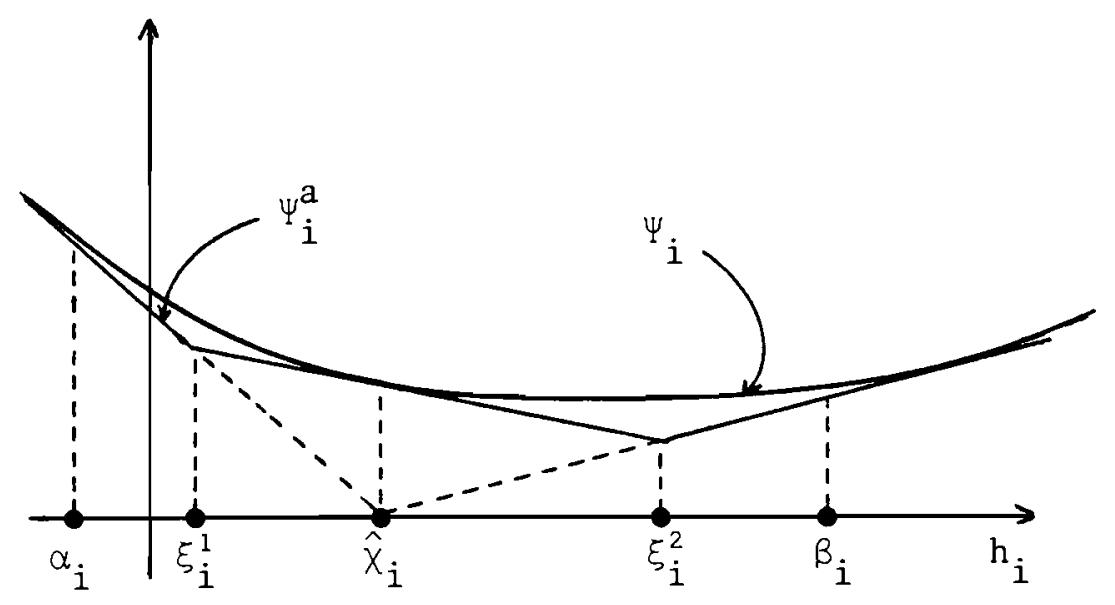

4.9. Figure: The function $\Psi^{\mathrm{a}}$

If the interval $\left[\alpha_{i}, \beta_{i}\right]$ has already been partitioned in $v$ intervals $\left\{\left[\alpha_{i}^{0}=\alpha_{i}, \alpha_{i}^{1}\right), \ldots,\left[\alpha_{i}^{\nu-1}, \alpha_{i}^{\nu}=\beta_{i}\right]\right\}$ and $\hat{x}_{i} \in\left[\alpha_{i}^{\ell}, \alpha_{i}^{\ell+1}\right)$. Then again the optimal subdivision of the interval $\left[\alpha_{i}^{l}, \alpha_{i}^{l+1}\right)$ into $\left[\alpha_{i}^{l}, \hat{x}_{i}\right)$ and $\left[\hat{x}_{i}, \alpha_{i}^{l+1}\right.$ ) yields an exact bound for $\Psi_{i}$ at $\hat{\chi}_{i}$. An alternative is to split the interval under consideration around $\hat{x}_{i}$ such that $\hat{x}_{i}$ turns out to be the conditional expectation of the new region. This would provide a quite good bound for $\Psi_{i}$ in the neighborhood of $\hat{x}_{i}$ and this would be very useful if the value of $x_{i}$ is not expected to change much in the next iterations.

4.10 IMPLEMENTATION. General recourse matrix $\mathrm{W}$, with $\mathrm{h}$ stochastic; $\mathrm{q}$ and $\mathrm{T}$ are fixed. The function

$$
h \mapsto \psi(x, h)
$$

is not separable, it is convex and polyhedral (1.11). Note also that 


$$
h \mapsto \psi(x, h-\chi)
$$

is a sublinear function. Because of this we shall say that $\psi\left(\chi_{,} \cdot\right)$ is sublinear with root at $\chi$. We assume that $\Xi \subset R^{m_{2}}$ is a rectangle and that we are given a partition $\left\{s^{\ell}, \ell=1, \ldots, \nu\right\}$ illustrated below.

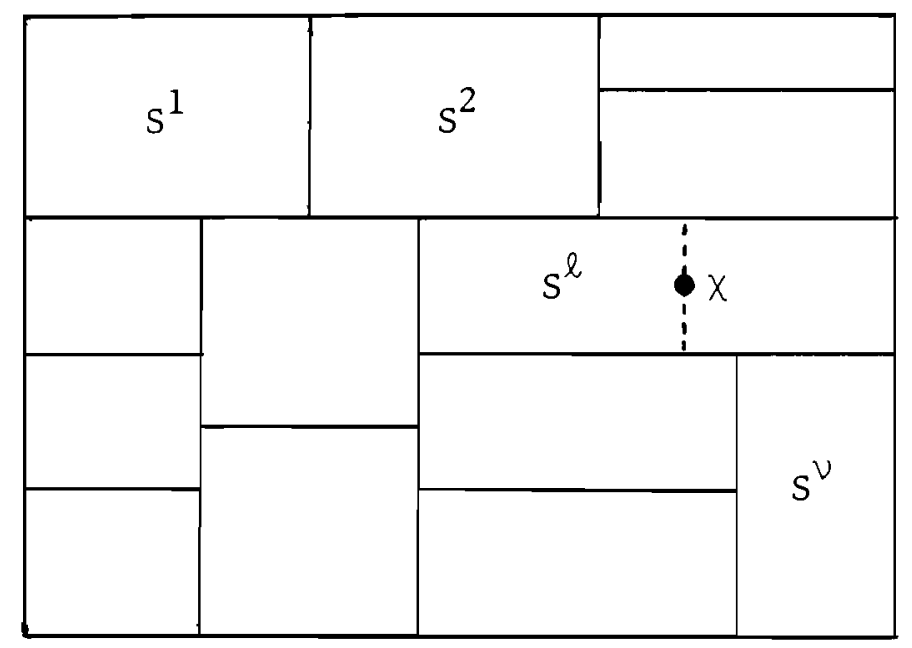

4.11. Figure: Partition $S=\left\{S^{1}, \ldots, S^{\nu}\right\}$ of $\Xi$

We shall take it for granted that the next partition of $\Xi$ will be obtained by splitting one of the cells $s^{\ell}$. Other partitioning strategies may be used but this single cell approach has the advantage of increasing only marginally the linear program that needs to be solved in order to obtain the lower bound.

(i) Let us first consider the case when $\chi \in S^{l} \subset \Xi$. We plan to split $S^{l}$ with a hyperplane containing $\chi$ and parallel to a face of $S^{\ell}$, or equivalently parallel to a hyperplane bounding the orthants. To do this, we study the behavior of $h \mapsto \psi(x, h)$ on each edge $E_{k}$ of the cell $s^{l}$. Let

$$
h \mapsto \theta_{k}(h)=\psi(x, h): E_{k} \rightarrow R .
$$

This is a piecewise linear convex function. The possible shape of this function is illustrated in Figure 4.12 below; by $\chi^{\mathrm{p}}$ we denote the orthogonal projection of $x$ on $\mathrm{E}_{\mathrm{k}}$. 

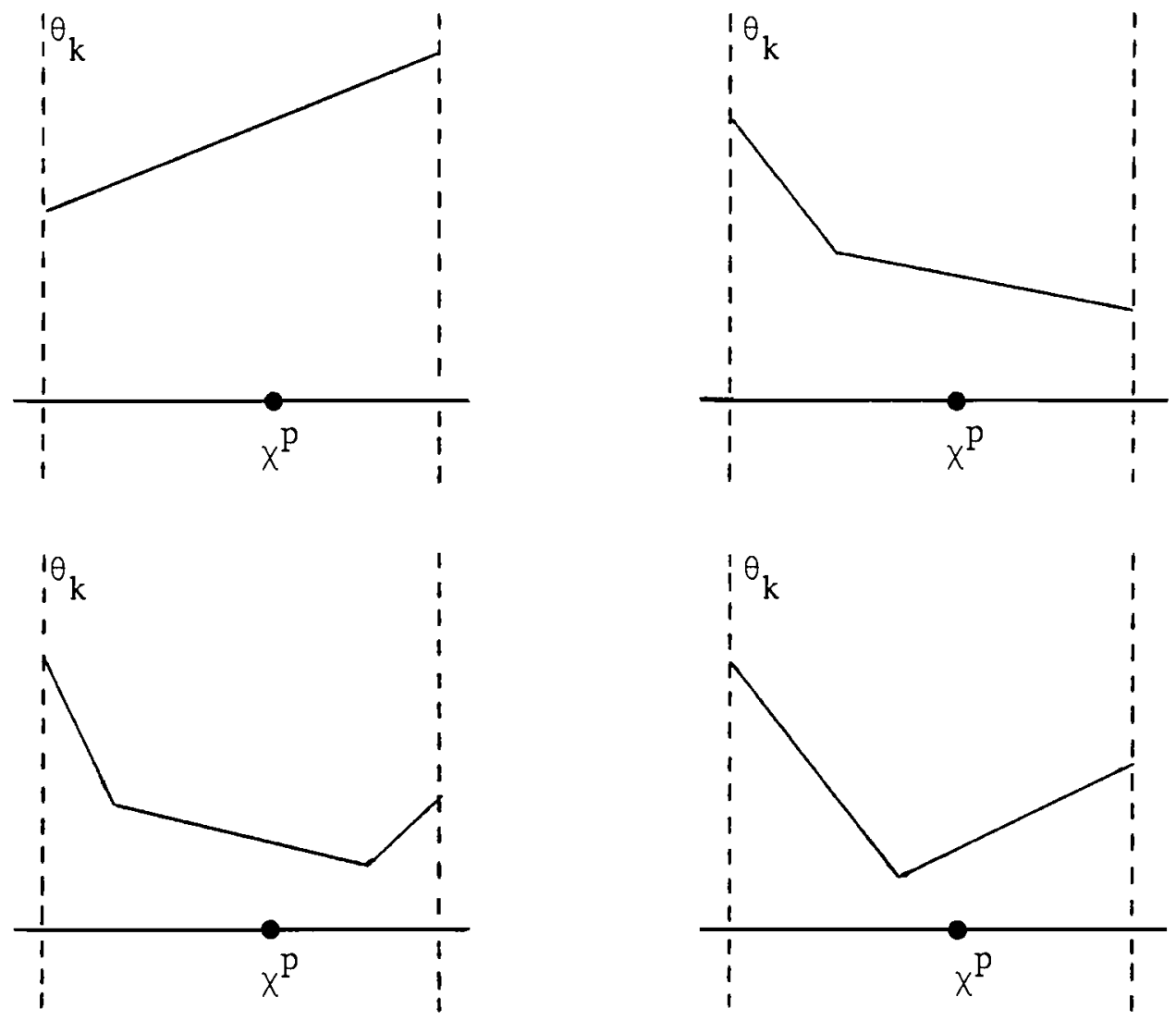

4.12. Figure: The function $\theta_{k}$ on $E_{k}$

If $\theta_{k}$ is linear on $E_{k}$, it means that we cannot improve the approximation to $\theta_{k}$ by splitting $S^{l}$ so as to subdivide $E^{k}$. On the contrary if the slopes of $\theta_{k}$ at the end points are different, then splitting $S^{l}$ so as to subdivide $E_{k}$ would improve the approximation to $\Psi$. On the subdivided cells, the resulting functions $\theta_{k}$ would be close to, if not actually, linear. Among all edges $E_{k}$, we would then choose to partition the cell $s^{\ell}$ so as to subdivide the edge $E_{k}$ that exhibits for $\theta_{k}$ the largest difference of slopes at the end points. What we need to know are the subgradients of the function

$$
\mathrm{h} \mapsto \psi(\chi, h)
$$

at each vertex $\left\{h^{s}, s=1, \ldots, r\right\}$ of the cell $s^{l}$. This is obtained by solving the linear programs 
(4.13) find $\pi \in R^{m_{2}}$ such that $\pi W \leq q$ and $w_{S}=\pi\left(h^{s}-X\right)$ is maximized

for $s=1, \ldots, r$. The optimal $\pi^{s}$ is a subgradient of $\psi(\chi, \cdot)$ at $h^{s}[1$, Proposition 7.12$]$. From this we obtain the directional subderivative of $\psi(\chi, \cdot)$ in each coordinate direction (which are the slopes of the functions $\theta_{k}$ ); they are simply the components of the vector $\left\{\pi_{i}^{s}, i=1, \ldots, m_{2}\right\}$. We now construct a subdivision of $s^{l}$ with a hyperplane passing through $x$ and orthogonal to the edge of $s^{l}$ that exhibits maximum slope difference. If the underlying probability structure is such that the random vector $h(\cdot)$ is the sum of a few random variables, such as described by (1.19), the calculation of the directional subderivatives of $\xi \leftrightarrow \psi(X, \xi)$ again begins with the calculation of the optimal solution of the programs (4.13) each $h^{s}$ being obtained as the map of a vertex of $s^{l}$ through the map (1.19). To obtain the subderivatives, we again need to use this transformation.

(ii) We now consider the case when $\chi \notin \Xi$. This time we cannot always choose a hyperplane passing through $\chi$ that generates a further subdivision of some cell $\mathrm{s}^{\ell}$. Even when this is possible, it might not necessarily improve the approximation, the function $\xi \mapsto \psi(\chi, \xi)$ being linear on that cell for example. Ideally, one should then search all cells $\mathrm{S}^{l}$ and each edge in any given cell to find where the maximum gain could be realized. Generally, this is impractical. What appears reasonable is to split the cell with maximum probability, on which $\psi(x, \cdot)$ is not linear.

Concerning the implementation of this partitioning technique, we are seeking the approximation to $\Psi$ which is as good as possible in the neighborhood of $\chi$. We are thus working with the implicit assumption that we are in a neighborhood of the optimal solution and that $\chi$ will not change significantly from one interaction to the next. If this is the case, and the problem is well-posed, then we 
shouldn't really have to deal with case (ii), since it would mean that the optimal tender $\chi^{0}$ would be such that we would consistently underestimate or overestimate the demand!

4.14 APPLICATION. Consider the stochastic program with recourse with only $q$ stochastic. With $S=\left\{S^{\ell}, \ell=1, \ldots, \nu\right\}$ a partition of $\Xi$, and for $\ell=1, \ldots, \nu$, let

$$
\xi^{\ell}=q^{\ell}=E\left\{q(\omega) \mid s^{\ell}\right\}
$$

and $p_{\ell}=P\left[\xi(\omega) \in S^{\ell}\right]$. As follows from (1.12) and (4.3) we have

$$
\text { (4.15) } \quad \sum_{\ell=1}^{\cup} \mathrm{p}_{\ell} \mathrm{Q}\left(\mathrm{x}, \xi^{\ell}\right) \geq 2(\mathrm{x}) \text {. }
$$

Thus, with

$$
z^{\nu}=\inf { }_{x \in R^{n_{1}}}\left[c x+\sum_{\ell=1}^{\cup} p_{\ell} Q\left(x, \xi^{\ell}\right) \mid A x=b, x \geq 0\right]
$$

where

$$
Q\left(x, \xi^{l}\right)=\inf _{y \in R^{n_{2}}}\left[q^{l} y \mid W y=h-T x, y \geq 0\right],
$$

we have that

$$
z^{\nu} \geq z^{*}=\inf [c x+2(x) \mid A x=b, x \geq 0] .
$$

Each $z^{\nu}$ is thus an upper bound for the optimal value of the stochastic program.

\subsection{IMPLEMENTATION. The function}

$$
\mathrm{q} \mapsto \psi(\chi, \mathrm{q})=\max \{\pi(\xi-\chi) \mid \pi W \leq \mathrm{q}\}
$$

is polyhedral and suplinear. What changes from one $x$ to the next are the slopes of this function, so we cannot use the present $\chi$ as a guide for the design of the approximation. One possibility in this case is to simply subdivide a cell of the partition with maximum probability. 


\section{DISCRETE PROBABILITY MEASURES WITH EXTREMAL SUPPORT}

The maximum of a convex function on a compact convex set is attained at an extreme point; moreover, the function value at any point (of its domain) obtained as a convex computation of extreme points is dominated by the same convex combination of the function values at those extreme points. These elementary facts are used in the construction of measures that yield upper bounds for the expectation functional $E_{\mathrm{f}}$.

5.1 PROPOSITION. Suppose $\xi \mapsto f(x, \xi)$ is convex, $\Xi$ the support of the random variable $\xi(\cdot)$ is compact, and let ext $\Xi$ denote the extreme points of co $\Xi$, the convex hull of $\Xi$. Suppose moreover that for all $\xi, \nu(\xi, \cdot)$ is a probability measure defined on (ext $\Xi, E)$ with $E$ the Borel field, such that

$$
\int_{\text {ext }} \mathrm{e} v(\xi, \mathrm{de})=\xi,
$$

and

$$
\omega \mapsto \nu(\xi(\omega), A)
$$

is measurable for all $\mathrm{A} \in E$. Then

$$
E_{f}(x) \leq \int_{\text {ext } \Xi} f(x, e) \lambda(d e)
$$

where $\lambda$ is the probability measure on $E$ defined by

$$
\lambda(\mathrm{A})=\int_{\Omega} v(\xi(\omega), \mathrm{A}) \mathrm{P}(\mathrm{d} \omega) .
$$

PROOF. The convexity of $f(x, \cdot)$ implies that for the measure $v$ as defined above

$$
f(x, \xi) \leq \int_{\operatorname{ext} \Xi} f(x, e) \vee(\xi, d e)
$$

Substituting $\xi(\cdot)$ for $\xi$ and integrating both sides with respect to $\mathrm{P}$ yields the desired inequality (5.2). 
5. 4 COROLLARY. Suppose $\xi \mapsto f(x, \xi)$ is convex, $\Xi$ the support of the random variable $\xi(\cdot)$ is compact, and let ext $\Xi$ denote the extreme points of co $\Xi$, the convex huzz of $\Xi$. Then

$$
E_{f}(x) \leq \max e \in \operatorname{ext} \Xi f(x, e)=f\left(x, e_{x}\right)
$$

PRO0F. Simply follows from $f\left(x, e_{x}\right) \geq f(x, \xi)$ for all $\xi \in \equiv$, or use Proposition 5.1 with $v$ concentrated on $e_{x}$.

5.6 APPLICATION. Consider the stochastic program with recourse (1.6) with only $h$ and $\mathrm{T}$ stochastic. Let us assume that $\Xi$ the support of the random variables $h(\bullet)$ and $T(\cdot)$ is compact, with

$$
\operatorname{ext} \Xi=\left\{\xi^{\ell}=\left(h^{\ell}, T^{\ell}\right), \quad l=1, \ldots, L\right\}
$$

the extreme points of co $\Xi$. We explicitly assume that $L$ is finite. As usual

$$
Q\left(x, \xi^{l}\right)=\min \left\{q y \mid W y=h^{l}-T^{l} x, y \geq 0\right\} .
$$

Then with

$$
\xi^{S} \in \operatorname{argmax}\left\{Q\left(x, \xi^{l}\right), \ell=1, \ldots, L\right\},
$$

as follows from (5.5), we have that

$$
Q(x) \leq Q\left(x, \xi^{s}\right)
$$

and thus

$$
\begin{aligned}
z^{*} & =\inf \{c x+2(x) \mid A x=b, x \geq 0\} \\
& \leq \inf \left\{c x+q y \mid A x=b, T^{s} x+W y=h^{s}, x \geq 0, y \geq 0\right\} .
\end{aligned}
$$

This is a very crude bound that can easily be improved by partitioning $\Xi$. Say $S=\left\{S^{k}, k=1, \ldots, \nu\right\}$ is a partition and for each $k$ we compute $\xi^{k} \in \operatorname{argmax}_{\xi \in S^{k}} Q(x, \xi)$.

Then

$$
Q(x)=\sum_{k=1}^{v} \int_{S} Q(x, \xi(\omega)) P(d \omega) \leq \sum_{k=1}^{U} \int_{S} Q\left(x, \xi^{k}\right) P(d \omega)
$$


with $\mathrm{p}_{\mathrm{k}}=\mathrm{P}\left[\xi(\omega) \in \mathrm{S}^{\mathrm{k}}\right]$, we obtain

$$
z^{*} \leq \inf \left\{c x+\sum_{k=1}^{v} p_{k} q y^{k} \mid A x=b, T^{k} x+w y^{k}=h^{k}, x \geq 0, y^{k} \geq 0\right\} .
$$

The potential use of this inequality as an approximation tool for solving stochastic programs with recourse was pointed out by Kall and Stoyan [18].

5.9 APPLICATION. We take the same situation as in Application 5.6. Let us define a probability measure $v(\xi, \cdot)$ on ext $\Xi=\left\{\xi^{1}, \ldots, \xi^{L}\right\}$, i.e. scalars $\left\{\mathrm{p}_{1}(\xi), \ldots, \mathrm{p}_{\mathrm{L}}(\xi)\right\}$ such that $\sum_{\ell=1}^{\mathrm{L}} \mathrm{p}_{\ell}(\xi)=1$ and

$$
\xi=(h, T)=\sum_{\ell=1}^{L} \mathrm{p}_{\ell}(\xi) \cdot\left(\mathrm{h}^{\ell}, \mathrm{T}^{\ell}\right)
$$

Then

$$
\mathrm{Q}(\mathrm{x}, \xi) \leq \sum_{\ell=1}^{\mathrm{L}} \mathrm{p}_{\ell}(\xi) \mathrm{Q}\left(\mathrm{x}, \xi^{\ell}\right)
$$

and

$$
Q(x) \leq \sum_{\ell=1}^{L} \bar{p}_{\ell} Q\left(x, \xi^{\ell}\right)
$$

where for each $\ell=1, \ldots, L$

$$
\overline{\mathrm{p}}_{\ell}=\int \mathrm{p}_{\ell}(\xi(\omega)) \mathrm{P}(\mathrm{d} \omega)
$$

The $\left\{\overline{\mathrm{p}}_{\ell}, \ell=1, \ldots, \mathrm{L}\right\}$ determine a probability measure on ext $\Xi$. The right-hand term of (5.11) may however be quite difficult to compute since the dependence of the $\mathrm{p}_{\ell}$ on $\xi$ may not be easy to express: they must be chosen so as to satisfy (5.10). There are some important cases when all of this can be worked out relatively easily. We review them next.

(i) $\Xi$ (or co $\Xi$ ) is a simplex. Each $\xi$ in $\Xi$ has a unique representation in terms of the extreme points ext $\Xi$, viz. in terms of its barycentric coordinates. For example, if co $\Xi$ is the fundamental simplex in $\mathrm{R}^{\mathrm{N}}$ whose extreme points are

$$
\left\{0,\left(e^{j}, j=1, \ldots, N\right)\right\} \quad \text { with } \quad e^{j}=(0, \ldots, 0,1,0, \ldots, 0)^{T} \text {, }
$$


then each point

$$
\xi=\left(\xi_{1}, \ldots, \xi_{N}\right) \text { in } \Xi
$$

has the barycentric representation

$$
\mathrm{p}_{\mathrm{o}}(\xi)=1-|\xi|, \quad\left(\mathrm{p}_{\ell}=\xi_{\ell}, \ell=1, \ldots, \mathrm{N}\right)
$$

where $|\xi|=\sum_{\ell=1}^{N} \xi_{\ell}$. Al1 other cases can be brought back to this one by an invertible linear transformation.

(ii) $\Xi$ is an interval. This is a special case of the preceding one. Let $\Xi=[\alpha, \beta]$, then $\xi=(1-p) \alpha+p \beta$ with $0 \leq p \leq 1$; and thus $p=(\xi-\alpha) /(\beta-\alpha)$ from which it follows that

$$
(1-\bar{p})=(\beta-\bar{\xi}) /(\beta-\alpha) \quad \text { and } \quad \bar{p}=(\bar{\xi}-\alpha) /(\beta-\alpha)
$$

with $\bar{\xi}=E\{\xi\}$. Thus

$$
Q(x) \leq(1-\bar{p}) Q(x, \alpha)+\bar{p} Q(x, \beta)
$$

This inequality is due to Edmundson. Madansky [12] used it in the context of stochastic programs with simple recourse random right-hand sides $h$. A much refined version of this upper bound can be obtained by partitioning the interval $[\alpha, \beta]$ and computing for each subinterval the corresponding version of (5.12). The expression for the $\overline{\mathrm{p}}$ will now involve conditional expectations. For stochastic programs with simple recourse this was carried out by Huang, Ziemba, and Ben-Tal [19] and by Kall and Stoyan [18] .

(iii) $\Xi$ is a rectangle and $\xi \mapsto Q(x, \xi)=\sum_{i=1}^{N} Q_{i}\left(x, \xi_{i}\right)$ is separable. This is the case of stochastic programs with simple recourse with stochastic $h$, for example. Then

$$
Q(x)=\sum_{i=1}^{N} \int Q_{i}\left(x, \xi_{i}(\omega)\right) P(d \omega) .
$$

We can now find bounds for $Q$ by seeking bounds for each $Q_{i}(x, \cdot)$ separately. 
We are in the situation considered in (ii). The inequality (5.12) becomes

$$
2(x) \leq \sum_{i=1}^{N}\left(1-\bar{p}_{i}\right) Q_{i}\left(x, \alpha_{i}\right)+\bar{p}_{i} Q_{i}\left(x, \beta_{i}\right)
$$

where $\left[\alpha_{i}, \beta_{i}\right]$ is the support of the random variable $\xi_{i}(\cdot)$ and $\bar{p}_{i}$ defined as above.

(iv) $\Xi$ is a rectangle and the random variables are independent. Let $F_{i}: R \rightarrow[0,1]$ be the distribution function of the random variable $\xi_{i}$. We have that

$$
Q(x)=\int_{\alpha_{N}}^{\beta_{N}} d F_{N}\left(\xi_{N}\right) \ldots . \int_{\alpha_{1}}^{\beta_{1}} d F_{1}\left(\xi_{1}\right) Q\left(x,\left(\xi_{1}, \ldots, \xi_{N}\right)\right)
$$

where $\Xi=x_{i=1}^{N}\left[\alpha_{i}, \beta_{i}\right]$. With $\xi_{2}, \ldots, \xi_{N}$ fixed, for each $\xi_{1} \in\left[\alpha_{1}, \beta_{1}\right]$, it follows from convexity that

$$
\begin{aligned}
Q\left(x,\left(\xi_{1}, \xi_{2}, \ldots, \xi_{N}\right)\right) \leq & \frac{\beta_{1}-\xi_{1}}{\beta_{1}-\alpha_{1}} Q\left(x,\left(\alpha_{1}, \xi_{2}, \ldots, \xi_{N}\right)\right. \\
& +\frac{\xi_{1}-\alpha_{1}}{\beta_{1}-\alpha_{1}} Q\left(\beta_{1}, \xi_{2}, \ldots, \xi_{N}\right) .
\end{aligned}
$$

Integrating on both sides with respect to $\mathrm{dF}_{1}$, and with $\bar{\xi}_{1}=\mathrm{E}\left\{\xi_{1}\right\}$ we have

$$
\begin{aligned}
\int_{\alpha_{1}}^{\beta_{1}} \mathrm{dF}\left(\xi_{1}\right) Q\left(x,\left(\xi_{1}, \xi_{2}, \ldots, \xi_{N}\right)\right) \leq & \frac{\beta_{1}-\bar{\xi}_{1}}{\beta_{1}-\alpha_{1}} Q\left(x,\left(\alpha_{1}, \xi_{2}, \ldots, \xi_{N}\right)\right) \\
& +\frac{\bar{\xi}_{1}-\alpha_{1}}{\beta_{1}-\alpha_{1}} Q\left(x,\left(\beta_{1}, \xi_{2}, \ldots, \xi_{N}\right)\right) .
\end{aligned}
$$

We can now repeat this process for $\xi_{2}$, considering the two functions

$$
\begin{aligned}
& \xi_{2} \mapsto Q\left(x,\left(\alpha_{1}, \xi_{2}, \ldots, \xi_{\mathrm{N}}\right),\right. \\
& \xi_{2} \mapsto Q\left(x,\left(\beta_{1}, \xi_{2}, \ldots, \xi_{N}\right) .\right.
\end{aligned}
$$

One obtains 


$$
\begin{aligned}
& \int_{\alpha_{2}}^{\beta_{2}} \mathrm{~d} F_{2}\left(\xi_{2}\right) \int_{\alpha_{1}}^{\beta_{1}} \mathrm{dF} F_{1}\left(\xi_{1}\right) \mathrm{Q}\left(\mathrm{x},\left(\xi_{1}, \xi_{2}, \ldots, \xi_{N}\right)\right) \\
& \leq\left[\left(\beta_{1}-\alpha_{1}\right)\left(\beta_{2}-\alpha_{2}\right)\right]^{-1}\left[\left(\beta_{1}-\bar{\xi}_{1}\right)\left(\beta_{2}-\bar{\xi}_{2}\right) Q\left(x,\left(\alpha_{1}, \alpha_{2}, \ldots, \xi_{N}\right)\right)\right. \\
& \quad+\left(\beta_{1}-\bar{\xi}_{1}\right)\left(\bar{\xi}_{2}-\alpha_{2}\right) Q\left(x,\left(\alpha_{1}, \beta_{2}, \ldots, \xi_{N}\right)+\left(\bar{\xi}_{1}-\alpha_{1}\right)\left(\beta_{2}-\bar{\xi}_{2}\right) Q\left(x,\left(\beta_{1}, \alpha_{2}, \ldots, \xi_{N}\right)\right)\right. \\
& \left.\quad+\left(\bar{\xi}_{1}-\alpha_{1}\right)\left(\bar{\xi}_{2}-\alpha_{2}\right) Q\left(x,\left(\beta_{1}, \beta_{2}, \ldots, \xi_{N}\right)\right)\right] .
\end{aligned}
$$

Doing this, in turn, for every $\xi_{3}, \ldots, \xi_{\mathrm{N}}$ yields an upper bound for 2 of the following type:

(5.14) $\quad Q(x) \leq \prod_{i=1}^{N}\left(\beta_{i}-\alpha_{i}\right)^{-1} \sum_{\gamma \in G}\left(\prod_{i=1}^{N}\left|\bar{\xi}_{i}-\gamma_{i}\right| Q\left(x,\left(\gamma_{1}, \ldots, \gamma_{N}\right)\right)\right)$

where $|\cdot|$ is absolute value, and $G$ is the collection of $2^{N}$ vectors defined by

$$
G=\left\{\gamma=\left(\gamma_{1}, \ldots, \gamma_{N}\right) \mid \gamma_{i}=\alpha_{i} \text { or } \beta_{i}, i=1, \ldots, N\right\}
$$

One can also interpret $(5.14)$ as follows: Let ext $\Xi=\left\{\xi^{\ell}, \ell=1, \ldots, L=2^{N_{N}}\right\}$ and now define on ext $\Xi$ a probability measure $\nu$ which assigns probability $\mathrm{p}_{\ell}$ to $\xi^{\ell}$, where

$$
p_{\ell}=\prod_{i=1}^{N}\left|\bar{\xi}_{i}-\xi_{i}^{\ell}\right| /\left(\beta_{i}-\alpha_{i}\right)
$$

Note that with this probability measure, suggested first in [18], yields an upper bound for 2 that does not require passing through a transformation assigning to each $\xi$ a particular combination of the extreme points.

(v) $\Xi$ is a polytope, possibly a rectangle. Let $S=\left\{S^{k}, k=1, \ldots, v\right\}$ be a simplicial decomposition of $\Xi$, i.e. the partition is generated by a complex whose cells are simplices. Then in each cell we are in the situation described in (i). On each one we have an upper bound of type (5.11) for

$$
\int_{\left\{\xi(\omega) \in S^{k}\right\}} Q(x, \xi(\omega)) P(d \omega)
$$


which we can then add up to obtain a bound for 2 . The bounds can be improved by refining the partition, for example. Another way is to consider for each $\xi$ not just one possible representation, but to look for the smallest upper bound given by a number of possible simplicial decompositions. Again, let $\left\{\xi^{\ell}, \ell=1, \ldots, L\right\}=\operatorname{ext} \Xi \subset R^{N}$, and $P$ the sets of all $N+1$ subsets of ext $\Xi$. Let $P(\xi)$ be the elements of $P$ such that $\xi$ belongs to their convex hull. Then

$$
Q(x, \xi) \leq \min \left\{\xi^{\ell_{0}}, \ldots, \xi^{\ell_{N}}\right\} \in P(\xi)\left\{\sum_{j=0}^{N} p_{j}(\xi) Q\left(x, \xi^{\ell_{j}}\right) \mid \sum_{j=0}^{N} p_{j}(\xi) \xi^{\ell_{j}}=\xi\right\} .
$$

Integrating on both sides, after replacing $\xi$ by $\xi(\omega)$, gives the desired upper bound on $2(x)$, and thus also on $z^{*}$ as defined by (5.7).

A last suggestion, in this general case, is for $\Xi$ a rectangle but the $\left\{\xi_{j}(\cdot), j=1, \ldots, N\right\}$ not independent. We still have that for all $j, \xi_{j} \mapsto Q(x, \xi)$ is convex. Set $j=1$. Using (5.5), and with $F$ the distribution function of $\xi(\bullet)$ on $\Xi$, we have that

$$
\begin{aligned}
& 2(x)=\int Q(x, \xi) d F(\xi)
\end{aligned}
$$

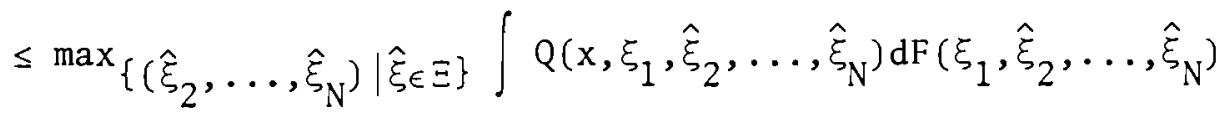

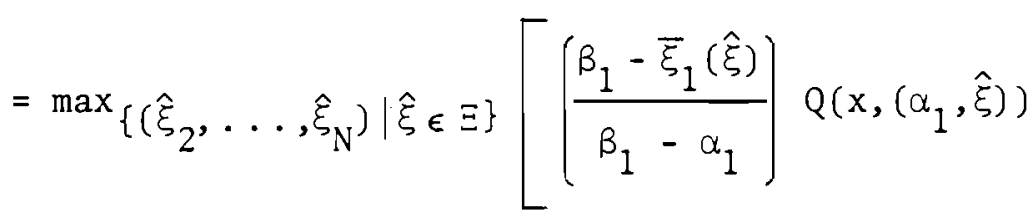

$$
\begin{aligned}
& \left.+\left(\frac{\bar{\xi}_{1}(\hat{\xi})-\alpha_{1}}{\beta_{1}-\alpha_{1}}\right) Q\left(x,\left(\beta_{1}, \xi\right)\right)\right]
\end{aligned}
$$

where $\bar{\xi}_{1}(\hat{\xi})$ is the conditional expectation of $\xi_{1}(\cdot)$ given $\hat{\xi}$. A bound of this type can be computed for each $j$ and then we should choose the smallest one to bound 2 . 
5.17 APPLICATION. Consider the stochastic program with recourse (1.15) with only $\mathrm{q}$ stochastic. If we now assume that $\Xi$, the support of the random variable $\xi(\cdot)=q(\cdot)$, is compact, all the bounds obtained for 2 when $h$ and $T$ are stochastic have their counterparts in this case, except that this time we get lower bounds instead of upper bounds.

5.18 IMPLEMENTATION. We are in the same situation as in Section 4 . Given a partition (simplicial decomposition, interval subdivision, or a rectangular cel1 splitting case such as illustrated by Figure 4.11) the question which arises is to find a refinement of the partition that adds only a few cells and improves the approximation as much as possible. In practice, this boils down, as in Implementation 4.10 , to subdividing just one cell. The piecewise linear character of $\xi \leftrightarrow Q(x, \xi)$ plays the predominant role; as a matter of fact, all the arguments used to justify subdivision by a hyperplane passing through $\chi$ still apply. We would thus follow the same strategies as those suggested in Implementations 4.7, 4.10 and 4.15 . The situation is illustrated by considering the simple recourse case (with $h$ stochastic). Then $h_{i} \mapsto \psi_{i}\left(X_{i}, h_{i}\right)$ is a 1-dimensional piecewise linear function. If $\left[\alpha_{i}, \beta_{i}\right]$ is the support of $h_{i}(\cdot)$, we have as a first bound

$$
\Psi_{i}\left(x_{i}\right) \leq \frac{\beta_{i}-\bar{h}_{i}}{\beta_{i}-\alpha_{i}} \psi_{i}\left(x_{i}, \alpha_{i}\right)+\frac{\bar{h}_{i}-\alpha_{i}}{\beta_{i}-\alpha_{i}} \psi_{i}\left(x_{i}, \beta_{i}\right) \text {. }
$$

Subdividing $\left[\alpha_{i}, \beta_{i}\right]$ at $x_{i}=\hat{\chi}_{i}$ we get

$$
\Psi_{i}\left(x_{i}\right) \leq \gamma_{\alpha} \psi_{i}\left(x_{i}, \alpha_{i}\right)+\hat{\gamma} \psi_{i}\left(x_{i}, \hat{x}_{i}\right)+\gamma_{\beta} \psi_{i}\left(x_{i}, \beta_{i}\right)
$$

where

$$
\begin{aligned}
& \gamma_{\alpha}=\left(\hat{x}_{i}-E\left\{h_{i}(\cdot) \mid h_{i}(\omega) \in\left[\alpha_{i}, \hat{x}_{i}\right)\right\}\right) /\left(\hat{x}_{i}-\alpha_{i}\right) \\
& \gamma_{\beta}=\left(E\left\{h_{i}(\cdot) \mid h_{i}(\omega) \in\left[\hat{x}_{i}, \beta_{i}\right]\right\}-\hat{x}_{i}\right) /\left(\beta_{i}-\hat{x}_{i}\right) \\
& \hat{\gamma}=\left(E\left\{h_{i} \mid\left[\alpha_{i}, \hat{x}_{i}\right)\right\}-\alpha_{i}\right)\left(\beta_{i}-E\left\{h_{i} \mid\left[\hat{x}_{i}, \beta_{i}\right]\right) /\left(\hat{x}_{i}-\alpha_{i}\right)\left(\beta_{i}-\hat{x}_{i}\right) .\right.
\end{aligned}
$$


This is a much tighter bound, with equality holding at $\hat{\chi}_{i}$. To illustrate what is going on, compare the graph of the approximating function $a_{1}$ to $\psi_{i}\left(x_{i}, \cdot\right)$ before subdividing at $\hat{\chi}_{i}$ and the graph of $a_{2}$ after subdivision.
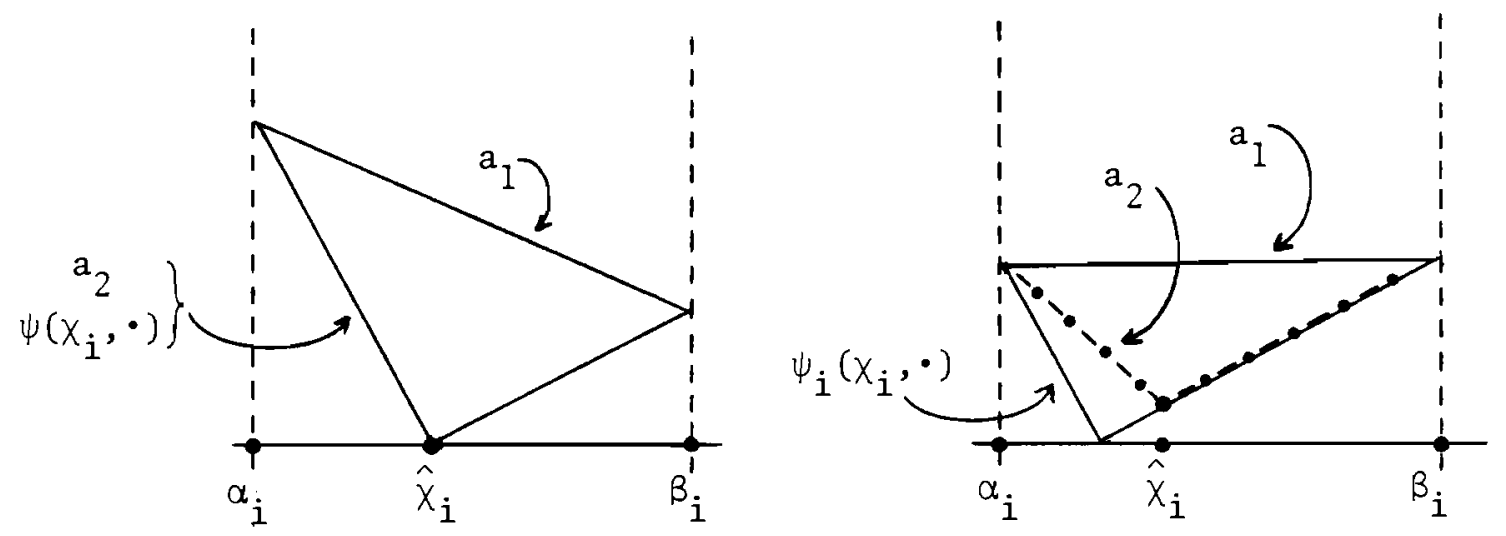

5.19. Figure: Splitting $\left[\alpha_{i}, \beta_{j}\right]$ at $\hat{x}_{i}$

5.19 CONVERGENCE. The same argument as that used to obtain Convergence 4.6 in Section 4, again relying on Theorem 2.8, applies to this case. Except here, we start with $\Xi$ compact and for all $\ell, \xi^{\ell}$ is the extreme point of the cell $S^{\ell}$ at which $\xi \leftrightarrow Q(x, \xi)$ attains its maximum or minimum, depending on $Q(x, \cdot)$ being convex or concave. 


\section{EXTREMAL PROBABILITY MEASURES}

The use of extremal measures to construct upper and lower bounds is intimately related to a number of questions usually raised in the context of stochastic optimization with partial or incomplete information. In order to find a bound for

$$
E_{f}(x)=\int f(x, \xi(\omega)) P(d \omega)
$$

we intend to replace $\mathrm{P}$ by another probability measure, say $\mathrm{P}_{V}$, that automatically guarantees

$$
E_{f}(x) \geq \int f(x, \xi(\omega)) P_{v}(d \omega)=E_{f}^{v}(x),
$$

or its converse. One way to do this is to find a measure $P_{V}$ in a certain class $P$ of probability measures on $(\Omega, A)$, which contains $P$, and that maximizes (or minimizes) the linear functional $P^{\prime} H \int f(x, \xi(\omega)) P^{\prime}(d \omega)$ on the set $P$. Since by assumption $P \in P$, we have

$$
\inf _{P^{\prime} \in P} \int f(x, \xi(\omega)) P^{\prime}(d \omega) \leq E_{f}(x) \leq \sup _{P^{\prime} \in P} \int f(x, \xi(\omega)) P^{\prime}(d \omega) .
$$

Note that the measures that minimize or maximize the preceding expressions in general depend on $x$, but not always. And if they do, quite often the same measure remains extremal for a relatively large neighborhood of $x$.

To exploit (6.2) in the search of upper and lower bounds for stochastic programming problems, the choice of $P$ is of utmost importance. On one hand we want $P$ to be "narrow" enough to give us a measure in the immediate neighborhood of P; on the other hand, the chosen measure $P_{v}$ should be such that finding $E_{f}^{V}(x)$ is easy. In the context of the applications we have in mind, this means that $P_{v}$ should be a discrete measure. One possibility is to define $P$ as a neighborhood of $P$ such as

$$
P=\left\{\text { prob. meas. } Q\left|\sup _{A \in A}\right| P(A)-Q(A) \mid \leq \varepsilon\right\},
$$


or even

(6.4) $P=\left\{\right.$ prob. meas. $\left.Q\left|\sup _{\theta \in \Theta}\right| \int \theta(\omega) P(d \omega)-\int \theta(\omega) Q(d \omega) \mid \leq \varepsilon\right\}$,

where $\Theta$ is a class of test functions. Or with $F$ the distribution function of $\xi(\cdot)$ defined on $\mathrm{R}^{\mathrm{N}}$

$$
P=\left\{\text { dist. funct. } G\left|\sup _{z}\right| F(z)-G(z) \mid \leq \varepsilon\right\}
$$

The class $P$ can be further restricted by limiting the acceptable class of measures to those having finite molecular support, etc. .

The construction of bounds through extremal measures will however follow a quite different course: $P$ will be defined by a finite number of equalities and inequalities which lead automatically to extremal measures with finite support. For a number of reasons that will become apparent later on, it is easier to work here with $\Xi \subset R^{N}$ as the support of the measure $P$ (technically, $\xi(\cdot)$ is then the identity map and $\Omega=\Xi)$. So let $P$ be the set of probability measures $Q$ on $\left(\Xi, B^{N}\right)$ that satisfy

$$
\int_{\Xi} v_{i}(\xi) Q(d \xi) \leq \alpha_{i}, \quad i=1, \ldots, s,
$$

$$
\int_{\Xi} v_{i}(\xi) Q(d \xi)=\alpha_{i}, \quad i=s+1, \ldots, M,
$$

where $M$ is finite and the $v_{i}$ are bounded continuous functions. We shall always assume that $P \in P$, i.e. satisfies the relations (6.6) and (6.7). The problem of finding a measure that satisfies these conditions and maximizes or minimizes

$$
\int_{\Xi} v_{o}(\xi) Q(d \xi)
$$

where $v_{0}(\xi)=f(x, \xi)$, can be viewed as a generalized moment problem [20]. For problems of this type, we have the following general result: 
6.9 THEOREM. Suppose $\Xi$ is compact. Then the set $P$ is convex and compact (with respect to the weak* topology), and $P=\mathrm{cl} c \mathrm{co}(\operatorname{ext} P)$. Assuming that $\mathrm{v}_{\mathrm{o}}$ is continuous relative to $\Xi$, then $Q \mapsto S v_{0}(\xi) Q(\mathrm{~d} \xi)$ attains its optimum (maximum or minimum) at an extreme point of $P$. Moreover the extremal measures of $P$ are precisely those having finite (molecular) support $\left\{\xi^{1}, \ldots, \xi^{\mathrm{L}}\right\}$ with $\mathrm{L} \leq \mathrm{M}+1$ such that the vectors

$$
\left[\begin{array}{c}
\mathrm{v}_{1}\left(\xi^{1}\right) \\
\vdots \\
\mathrm{v}_{\mathrm{M}}\left(\xi^{1}\right) \\
1
\end{array}\right], \cdots,\left[\begin{array}{c}
\mathrm{v}_{1}\left(\xi^{\mathrm{L}}\right) \\
\vdots \\
\mathrm{v}_{\mathrm{M}}\left(\xi^{\mathrm{L}}\right) \\
1
\end{array}\right]
$$

are linearly independent.

Except for the presence of inequalities in the definition of $P$, this result can be found in [21, Theorem 2.1]. Dupačova, whose minimax approach to stochastic programming [22] led to the investigation of extremal measures was first in obtaining results of this type [23],[24] for a more classical formulation of the moment problem. The proof we give here, based on generalized linear programming [25, Chap.24], is due to Ermoliev, Gaivoronsky and Nedeva [26]. The reason for reproducing it here is that it is constructive and used in the sequel.

PRO0F. Suppose the constraints $(6.6),(6.7)$, and

$$
\int \mathrm{Q}(\mathrm{d} \xi)=1
$$

are consistent, otherwise there is nothing to prove. The convexity of $P$ is easy to verify, the compactness for the space of measures on a compact metric space follows from Prohorov's Theorem, and $P=c 1$ co(ext $P$ ) from the Krein-Milman Theorem 
about the representation of the elements of a convex set as convex combinations of its extremal points.

Now suppose that $\left\{\xi^{1}, \ldots, \xi^{\nu}\right\}$ is a finite collection of points of $\Xi$ that we view as part of the potential support of the extremal measure that maximizes (6.8); the case of minimization of (6.8) involves the same arguments and does not need to be dealt with separately. The question now is to assign to these points $\left\{\xi^{1}, \ldots, \xi^{\nu}\right\}$ a probability distribution that maximizes (6.8). This can be expressed as a linear programming problem, with variables $\left\{\mathrm{p}_{1}, \ldots, \mathrm{p}_{\nu}\right\}$, formulated as follows:

(6.10) find $p_{1} \geq 0, \ldots, p_{V} \geq 0$ such that $\sum_{\ell=1}^{v} p_{\ell}=1$,

$$
\begin{aligned}
& \sum_{\ell=1}^{v} v_{i}\left(\xi^{\ell}\right) p_{\ell} \leq \alpha_{i}, \quad \text { for } i=1, \ldots, s \\
& \sum_{\ell=1}^{v} v_{i}\left(\xi^{\ell}\right) p_{\ell}=\alpha_{i}, \quad \text { for } i=s+1, \ldots, M \\
& \text { and } z=\sum_{\ell=1}^{v} v_{0}\left(\xi^{\ell}\right) p_{\ell} \text { is maximized. }
\end{aligned}
$$

Assuming the points $\left\{\xi^{1}, \ldots, \xi^{\nu}\right\}$ have been picked so as to make this problem feasible, it is then also solvable. Let $\left\{p_{\ell}^{v}, \ell=1, \ldots, \nu\right\}$ denote the optimal solution and let

$$
\left(\theta^{\nu}, \pi_{1}^{\nu}, \ldots, \pi_{s}^{\nu}, \pi_{s+1}^{\nu}, \ldots, \pi_{M}^{\nu}\right)=\left(\theta^{\nu}, \pi^{\nu}\right)
$$

be the simplex multipliers associated at the optimum to the constraints of (6.10). The measure determined by

$$
Q_{v}\left[\xi^{\ell}\right]=p_{\ell}^{\nu}, \quad \ell=1, \ldots, v
$$

is the desired extremal measure, unless some $\xi$ in $\Xi$ can be found such that

(6.11) $v_{0}(\xi)-\sum_{i=1}^{M} \pi_{i}^{\nu} v_{i}(\xi)-\theta^{\nu}>0$. 
This follows directly from the optimality criteria for linear programs, when we note that each $\xi$ in $\Xi$ potentially generates a column that could be added to (6.10). If $(6.11)$ holds for some $\xi$, let

$$
\xi^{\nu+1} \epsilon \operatorname{argmax}\left[v_{0}(\xi)-\sum_{i=1}^{M} \pi_{i}^{\nu} v_{i}(\xi) \mid \xi \epsilon \Xi\right] \text {. }
$$

The existence of $\xi^{\nu+1}$ is not in question since the $v_{i}$ are continuous and $\Xi$ is compact. Adding the column

$$
\left[\begin{array}{c}
1 \\
v_{1}\left(\xi^{\nu+1}\right) \\
\vdots \\
v_{M}\left(\xi^{\nu+1}\right) \\
v_{0}\left(\xi^{\nu+1}\right)
\end{array}\right]
$$

to the linear program $(6.10)$ is guaranteed to yield a new solution $\left\{p_{\ell}^{\nu+1}, \ell=1, \ldots, \nu+1\right\}$ and a measure

$$
Q_{v+1}\left[\xi^{\ell}\right]=p_{\ell}^{\nu+1}, \quad \ell=1, \ldots, v+1
$$

such that

$$
\int v_{0}(\xi) Q_{v}(d \xi)<\int v_{0}(\xi) Q_{v+1}(d \xi)
$$

Repeating this until the point $\xi$ generated by (6.12) fails to satisfy (6.11) yields the extremal measure that maximizes $(6.8)$. Since this is generalized linear programming, the convergence proof of Dantzig [25, Chap.24] applies; a variant appears in [27, Chap.11.B] which can be exploited to obtain convergence of a modified procedure that only requires verification of (6.11) up to $\varepsilon$ [26, Theorem 5], a most desirable feature in practice.

To complete the proof of the Theorem, it suffices to observe that the optimal basis, associated with the solution of (6.10) will involve at most $M+1$ columns 
of the type $\left[1, v_{1}\left(\xi^{l}\right), \ldots, v_{M}\left(\xi^{l}\right)\right]^{T}$ that are linearly independent. And this holds for every possible objective $\int v_{0}(\xi) Q(d \xi)$, which by varying $v_{0}$ yields all extreme points of $P$.

Theorem 6.9 can now be applied to a variety of cases. The simplest one is when $\Xi=[\alpha, \beta] \subset R^{1}$, and the only condition is that the expectation with respect to $P_{V}$ should match the expectation $\bar{\xi}$ with respect to $P$. The problem reads:

find $Q$ a measure on $\left(\Xi, B^{1}\right)$ such that $Q \geq 0$,

$$
\begin{aligned}
& \int_{\alpha}^{\beta} \mathrm{Q}(\mathrm{d} \xi)=1, \int_{\alpha}^{\beta} \xi \mathrm{Q}(\mathrm{d} \xi)=\bar{\xi}, \\
& \text { and } \int_{\alpha}^{\beta} \mathrm{v}_{\mathrm{o}}(\xi) \mathrm{Q}(\mathrm{d} \xi) \text { is maximized . }
\end{aligned}
$$

Using the mechanism of the algorithm for generating $P_{V}$, in particular (6.12), it is not difficult to see that

$$
\text { with } v_{0} \text { concave, } \mathrm{P}_{v}\{\bar{\xi}\}=1 \text {, }
$$

(6.14) with $v_{0}$ convex, $P_{v}\{\alpha\}=\frac{\beta-\bar{\xi}}{\beta-\alpha}, P_{v}\{\beta\}=\frac{\bar{\xi}-\alpha}{\beta-\alpha}$.

This result and extensions thereof involving conditional expectation conditions, variance and unimodality conditions have been obtained and then applied to stochastic programming problems by Dupačova [23],[24], and Cipra [28]. Observe that the extremal measure defined by $(6.14)$ is precisely the discrete measure with extremal support obtained in Application 5.6 when $\Xi$ is an interval (Case (ii)) and $\xi \mapsto v_{0}(\xi)=Q(x, \xi)$ is convex. In fact, many of the results obtained in Sections 4 and 5 can be recovered by a judicious application of Theorem 6.9.

What limits the use of Theorem 6.9 in practice is solving (6.12)! In general, the function 
(6.15) $\quad \xi \mapsto v_{0}(\xi)-\sum_{i=1}^{M} \pi_{i}^{\nu} v_{i}(\xi)$

is neither convex nor concave, whatever be the properties of the $v_{i}$, since the $\pi_{i}^{v}$ are not restricted in sign. The remainder of this section is concerned with how to handle this global optimization problem in the context of stochastic programs with recourse. We begin with the simplest case.

6.16 APPLICATION. Consider the stochastic program with simple recourse with random right-hand sides $h$, i.e. of the form (1.15) with $\psi(\chi, \xi)$ defined through (3.21) and (3.22). The problem is then separable and the function $v_{0}$ can be expressed as

$$
v_{0}(\xi)=\sum_{j=1}^{m_{2}} v_{o j}\left(\xi_{j}\right)=\sum_{j=1}^{m_{2}} \psi_{j}\left(x_{j}, \xi_{j}\right)
$$

and consequently is also separable. Each generalized moment condition of type (6.6) or (6.7) would only involve the $\xi_{\mathrm{j}}(\cdot)$ separately (it would not make much sense otherwise). Thus finding the maximum (or the minimum) of $v_{0}-\sum_{i=1}^{M} \pi_{i}^{v} v_{i}$ is reduced to $\mathrm{N}\left(=\mathrm{m}_{2}\right)$ 1-dimensional maximization problems that can be handled in practice in a number of ways, see also [29].

\subsection{IMPLEMENTATION. We have to solve}

(6.18) find $\xi \in[\alpha, \beta]$ such that $z=v_{o j}(\xi)-\sum_{i=1}^{M} \pi_{i}^{v} v_{i j}(\xi)$ is maximized. We consider the case when $M=2, v_{i j}(\xi)=\xi, v_{2 j}(\xi)=\xi^{2}$; we want to match the first two moments. The function $\phi(\xi)=v_{o j}(\xi)$ is convex (1.11). Reformulating (6.18) we have:

(6.19) find $\xi \in[\alpha, \beta]$ such that $\phi(\xi)-\pi_{1}^{\nu} \xi-\pi_{2}^{\nu} \xi^{2}$ is maximized. If $\pi_{2}^{\nu} \leq 0$, the objective function is convex, in which case we only need to examine 
its values at the boundary points of the interval. If $\pi_{2}^{v}>0$, the interval can be divided up into regions of convexity and concavity and on each one the maximum can be found by conventional methods. Another possibility when higher moments are involved, is to use the bounds on the expected value of a convex function, obtained by Don [30], for a class of sample based probability measures. The optimal points of density $\xi^{1}, \ldots, \xi^{l}$ and the associated probabilities $\mathrm{p}_{1}, \ldots, \mathrm{p}_{\ell}$ are then straightforward to calculate provided the measure $P$ has certain symmetries. When this last condition is not satisfied, we could still use the so-generated discrete measure to initialize an algorithmic procedure for solving (6.18).

6.20 APPLICATION. Consider the stochastic program with recourse (1.15) with random right-hand sides $h$. Suppose

$$
S=\left\{\Xi_{i}, i=1, \ldots, M\right\}
$$

is a partition of $\Xi$, for every $i=1, \ldots, M, v_{i}$ is the indicator function of $\Xi_{i}$ and $\alpha_{i}=E\left\{h(\cdot) \mid \Xi_{i}\right\}$ is the conditional expectation of $h(\cdot)$ given $\Xi_{i}$. For $i=1, \ldots, M$, let

$$
p_{i}=P\left(\Xi_{i}\right)=P\left[h(\cdot) \epsilon \Xi_{i}\right]
$$

and again let $v_{0}(\xi)=\Psi(\chi, \xi)$. The problem of maximizing $v_{0}$ subject to (6.7) is then decomposable, in that each subregion $\Xi_{i}$ can be dealt with separately. Indeed,

$$
\int v_{0}(\xi) P(d \xi)=\sum_{i=1}^{M} \int_{\Xi_{i}} v_{0}(\xi) P(d \xi)
$$

and thus the original problem decomposes into solving $M$ subproblems of the type

(6.21) find a probability measure $Q_{i}$ on $\Xi_{i}$

$$
\begin{aligned}
& \text { such that } \int_{\Xi_{i}} \xi Q_{i}(d \xi)=\alpha_{i} \\
& \text { and } \int_{\Xi_{i}} v_{o}(\xi) Q_{i}(d \xi) \text { is maximized (or minimized). }
\end{aligned}
$$


With $\mathrm{P}_{i}^{\nu}$ the optimal solution to $(6.21)$, the desired measure is given by

$$
\mathrm{p}^{\nu}=\sum_{i=1}^{\mathrm{M}} \mathrm{p}_{i} \mathrm{P}_{i}^{\nu}
$$

Solving (6.21) is in principle not any easier than solving the general problem, except that we are only dealing with linear functions $v_{i}$ (which means that the convexity of $v_{0}$ yields the convexity of the objective function of the subproblem (6.15)) and if the partition $S$ of $\Xi$ is left to us, we can choose it so that it corresponds to linear pieces of $\xi \leftrightarrow \Psi(\chi, \xi)$.

\subsection{APPLICATION. Consider the stochastic program with recourse (1.15) with} random right-hand sides $h$, with the $h_{i}(\cdot)$ independent random variables for $i=1, \ldots, m_{2}$; we also have that $v_{0}(\xi)=\Psi(\chi, \xi)$. With the independence of the random variables comes the separability of the constraints (6.6) and (6.7). We would thus have a relatively easy problem to solve if it were not for the intricate relationship between the $\xi_{i}=h_{i}$ that appears in the objective $v_{0}$.

6.23 IMPLEMENTATION. If we are interested in the probability measure that minimizes $\int v_{0}(\xi) Q(d \xi)$ we can rely on the approximation to $\Psi$ provided by Application 3.3 . We have that

$$
v_{0}(\xi) \geq \max _{1 \leq \ell \leq L} \eta^{\ell}(\xi-\chi)
$$

where $x=T x$ and, as in Application 3.3,

$$
n^{\ell} \in \operatorname{argmax}\left[n\left(\xi^{\ell}-x\right) \mid \eta w \leq q\right]
$$

for $\left\{\xi^{\ell}=h^{\ell}, \ell=1, \ldots, L\right\}$ a finite number of realizations of $h(\cdot)$. Minimizing the function (6.15) that appears in the subproblem can then be expressed as find $\theta \in R_{1}$ and $\left(\xi_{i} \in \bar{\Xi}_{i}, i=1, \ldots, m_{2}\right)$ such that $\theta \geq n_{\ell}(\xi-x), \quad \ell=1, \ldots, L$, and $\theta-\sum_{i=1}^{M} \pi_{i}^{\nu} v_{i}(\xi)$ is minimized. 
If, for example, the functions $v_{i}$ correspond to first- and second-order moments, then this is a quadratic program, not necessarily convex. To solve it, we can rely on existing subroutines [31].

6.24 IMPLEMENTATION. If in Application 6.22, we are interested in the probability measure that maximizes $\int \Psi(\chi, \xi) Q(\mathrm{~d} \xi)$, we rely instead on the approximation to $\Psi(\bullet, X)$ which comes from Application 3.23 , which gives a separable function $v_{0}$, actually of the same type as for stochastic programs with simple recourse. This brings us to the case already studied in Application 6.16 and Implementation 6.17.

We note that the use of the approximating functions for the recourse function makes the calculation of extremal measures a reasonable undertaking, the global optimization problem of finding the $\xi \in \Xi$ that maximizes (or minimizes) the function $v_{0}(\cdot)-\sum_{i=1}^{M} \pi_{i}^{v} v_{i}(\cdot)$ would be too involved to solve exactly, just to obtain error bounds. Finally, we observe that all the results derived here could be extended to $h, T$ and $q$ stochastic; each case however requires a separate analysis to take full advantage of the properties of the problem under consideration. As more information is gathered about these types of approximation and resulting bounds, we expect to see a more detailed analysis of each case. The use of these techniques in an overall scheme for solving stochastic programs with recourse also needs further study in that here we have limited ourselves to finding extremal measures that yield the best possible lower and upper bounds for a given $x$ or $x$. Changing $x$ only affects the function $v_{0}$ and all that may be needed when passing from some $x$ to another is a recalculation of the weight factors $p_{1}, \ldots, p_{v}$ in (6.10), the points $\left\{\xi^{1}, \ldots, \xi^{\vee}\right\}$ remaining unchanged. Moreover at each new $x$, it may not be necessary to solve the generalized moment problem to optimality. 
6.25 CONVERGENCE. To obtain convergence, we need to consider sequences of generalized moment problems with an increasing number of restrictions on the moments of $\xi(\bullet)$. If this is done, we obtain a sequence of extremal measures $\left\{P_{v}, v=1, \ldots\right\}$ that converge in distribution to $\mathrm{P}$, and we are then in the setting of Theorem 2.8 . 


\section{MAJORIZING PROBABILITY MEASURES}

The role that convexity played in obtaining many of the bounds in the previous sections is taken over here by order preserving properties. The approximations are based on stochastic ordering [32, Chap.17]. They are especially useful because of their simple calculability. The use of majorizing measures to approximate stochastic programs was first advocated in [7].

We denote by $\precsim_{C}$ the partial ordering induced by the closed convex cone $\mathrm{C}$ on $R^{N}$. We write

$$
\mathrm{t}^{1} \precsim_{\mathrm{C}} \mathrm{t}^{2} \quad \text { if } \quad \mathrm{t}^{2}-\mathrm{t}^{1} \in \mathrm{C} \subset \mathrm{R}^{\mathrm{N}}
$$

and say that $t^{1}$ precedes $t^{2}$ (with respect to $\precsim_{C}$ ). A random vector $\xi^{1}: \Omega \rightarrow R^{N}$ stochastically precedes the random vector $\xi^{2}: \Omega \rightarrow R^{N}$ (with respect to $\precsim_{C}$ ) if

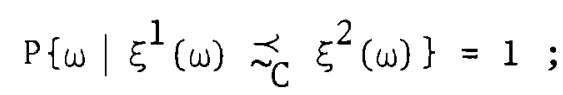

we write $\xi^{1}(\cdot) \underset{\sim_{C}}{\prec} \xi^{2}(\cdot)$. A function $\phi$ from $R^{N}$ into $R \cup\{+\infty\}$ is order preserving with respect to $\precsim_{\mathrm{C}}$ if

$$
\mathrm{t}^{1} \precsim_{\mathrm{C}} \mathrm{t}^{2} \quad \text { implies } \quad \phi\left(\mathrm{t}^{1}\right) \leq \phi\left(\mathrm{t}^{2}\right) .
$$

For $\phi$ order preserving and $\xi^{1}(\cdot) \precsim_{C} \xi^{2}(\cdot)$, obvious $1 y$

$$
E\left\{\phi\left(\xi^{1}(\omega)\right)\right\} \leq E\left\{\phi\left(\xi^{2}(\omega)\right)\right\}
$$

From this, it follows directly:

7.2 PROPOSITION. Suppose $\xi \mapsto f(x, \xi)$ is order preserving with respect to $\precsim_{C}$ and for $\mathrm{i}=1,2, \xi^{i}(\cdot):(\Omega, A, P) \rightarrow\left(R^{N}, B^{N}, F_{i}\right)$ are two random vectors such that $\xi^{1}(\cdot)$ stochastically precedes $\xi^{2}(\cdot)$. Then

$$
E_{f}^{1}(x)=\int f\left(x, \xi^{1}(\omega)\right) P(d \omega) \leq \int f\left(x, \xi^{2}(\omega)\right) P(d \omega)=E_{f}^{2}(x)
$$


7.4 APPLICATION. Consider the stochastic program with recourse (1.15) with only $h(\cdot)$, the right-hand sides, stochastic. Let

$$
\operatorname{pos} w=\left\{t \mid t=\sum_{j=1}^{n_{2}} w^{j} y_{j}, y_{j} \geq 0\right\},
$$

the convex cone generated by the columns of $W$, see $(1.17)$. Let $\left\{t^{\ell} \in \mathrm{R}^{\mathrm{m}_{2}}, \ell=1, \ldots, \mathrm{L}\right\}$ be a frame for this polyhedral cone, i.e. the vectors $t^{\ell}$ are positively linearly independent and pos $\left(t^{\ell}, \ell=1, \ldots, L\right)=$ pos $w$. Suppose that for all $\ell=1, \ldots, L$, and $\xi \in \Xi$, the function

$$
\lambda \mapsto \psi\left(x, \xi+\lambda t^{\ell}\right): R_{+} \rightarrow \bar{R}
$$

is monotone increasing. Then, if $\xi^{\mathrm{L}}(\cdot) \underset{\text { pos } w}{\aleph} \xi(\cdot)$

$$
\Psi^{\mathrm{L}}(x):=\int \psi\left(x, \xi^{\mathrm{L}}(\omega)\right) P(\mathrm{~d} \omega) \leq \Psi(X)
$$

and, if $\xi(\cdot) \precsim_{\text {pos } w} \xi^{\mathrm{U}}(\cdot)$

$$
\Psi(x) \leq \Psi^{U}(X):=\int \psi\left(x, \xi^{U}(\omega)\right) P(d \omega)
$$

This all follows directly from Proposition 7.2. It suffices to verify that the conditions imply that $\xi \rightarrow \psi(\chi, \xi)$ is order preserving with respect to $\precsim_{\text {pos } W}$, details are worked out in [7, Proposition 3.2].

Below, in Application 7.8, we give an example where the monotonicity of $\psi$ in each direction $t^{\ell}$ can be verified directly. In other cases, one may have to rely on various properties of the problem at hand. The construction of the random variables $\xi^{\mathrm{L}}(\cdot)$ and $\xi^{\mathrm{U}}(\cdot)$ relies on subdividing the range of $\xi(\cdot)$ into subsets generated by the partial ordering $\precsim_{\text {pos } w}$. This is done in [7, Section 3]. Convergence can be obtained by relying on finer and finer subdivisions of $\bar{E}$ and by relying on a special form of Theorem 2.8. We shall concentrate instead on questions of implementability and special cases. 
7.7 APPLICATION. Consider the stochastic program with recourse (1.15) with only $q(\cdot)$ stochastic. Let

$$
D(W)=\left\{y \mid y \geq \pi W \text { for some } \pi \in R^{m_{2}}\right\}
$$

and let $\left\{u^{\ell}, \ell=1, \ldots, L\right\}$ be a frame for the convex polyhedral cone $D(W)$. Suppose that for all $\ell=1, \ldots, L$ and $\xi \in \Xi$, the function

$$
\lambda \rightarrow \psi\left(x, \xi+\lambda u^{\ell}\right): R_{+}^{N} \rightarrow \bar{R}
$$

is monotone increasing, and

$$
\xi^{\mathrm{L}}(\cdot) \underset{\mathrm{D}(W)}{\precsim} \xi(\cdot) \underset{\mathrm{D}(W)}{\precsim} \xi^{\mathrm{U}}(\cdot)
$$

Then

$$
\text { (7.8) } \quad \Psi^{\mathrm{L}}(\chi):=\int \psi\left(x, \xi^{\mathrm{L}}(\omega)\right) \mathrm{P}(\mathrm{d} \omega) \leq \Psi(x)
$$

and

$$
\Psi(x) \leq \psi^{U}(x):=\int \psi\left(x, \xi^{U}(\omega)\right) P(d \omega)
$$

To apply Proposition 7.2 , we need to show that the monotonicity of $\lambda \mapsto \psi\left(x, \xi+\lambda u^{\ell}\right)$ for $\ell=1, \ldots, L$ implies that $\xi \mapsto \psi(X, \xi)$ is order preserving. Suppose $\xi^{1} \precsim_{D(W)} \xi^{2}$, then $\xi^{2}-\xi^{1} \in D(W)$ which means that

$$
\xi^{2}=\xi^{1}+\sum_{\ell=1}^{L} \alpha_{\ell} u^{\ell}
$$

for some scalars $\alpha_{\ell} \geq 0$. Relying on the monotonicity of $\psi$ in each coordinate, we obtain:

$$
\psi\left(x, \xi^{1}\right) \leq \psi\left(\chi, \xi^{1}+\alpha_{1} u^{1}\right) \leq \psi\left(x, \xi^{1}+\alpha_{1} u^{1}+\alpha_{2} u^{2}\right) \leq \cdots \leq \psi\left(x, \xi^{2}\right) .
$$

Note that $h$ and $q$ stochastic can be handled simultaneously provided naturally that the conditions laid out in Applications 7.4 and 7.7 be satisfied; this suggests some of the advantages of this approach. The real utility of this approach is, however, in the separable case. 
7.8 APPLICATION. Consider a stochastic program with simple recourse with random right-hand sides $h$, i.e. of the form (1.15) with $\psi(\chi, \xi)$ separable as defined by (3.21) and (3.22). Suppose that for $i=1, \ldots, m_{2}, q_{i}^{+} \geq 0$ and $q_{i}^{-} \geq 0$, and define $\xi_{i}^{\mathrm{L}}(\cdot)$ and $\xi_{i}^{\mathrm{U}}(\cdot)$ as follows:

$$
\begin{array}{ll}
\xi_{i}^{\mathrm{L}}(\cdot) \geq \xi_{i}(\cdot) & \text { on }\left\{\omega \mid \xi_{i}(\omega) \leq \chi_{i}\right\} \\
\xi_{i}^{L}(\cdot) \leq \xi_{i}(\cdot) & \text { otherwise }
\end{array}
$$

and

$$
\begin{array}{ll}
\xi_{\mathbf{i}}^{\mathrm{U}}(\cdot) \leq \xi_{\mathbf{i}}(\cdot) & \text { on }\left\{\omega \mid \xi_{\mathbf{i}}(\omega) \leq \chi_{\mathbf{i}}\right\} \\
\xi_{\mathbf{i}}^{\mathrm{U}}(\cdot) \geq \xi_{\mathbf{i}}(\cdot) & \text { otherwise. }
\end{array}
$$

Then

and

$$
\Psi^{L}(x):=\sum_{i=1}^{\mathrm{m}_{2}} \int \psi_{i}\left(\chi_{i}, \xi_{i}^{\mathrm{L}}(\omega)\right) P(\mathrm{~d} \omega) \leq \Psi(x),
$$

$$
\Psi(\chi) \leq \Psi^{U}(\chi):=\sum_{i=1}^{m_{2}} \int \psi_{i}\left(\chi_{i}, \xi_{i}^{U}(\omega)\right) P(d \omega)
$$

To see this, observe that the functions

$$
\xi_{i} \mapsto \psi_{i}\left(x_{i}, \xi_{i}\right)
$$

are monotone decreasing on $\left(-\infty, \chi_{i}\right]$, and monotone increasing on $\left[x_{i},+\infty\right)$ since

$$
\psi_{i}\left(x_{i}, \xi_{i}\right)=\left[\begin{array}{ccc}
q_{i}^{-}\left(x_{i}-\xi_{i}\right) & \text { if } & \xi_{i} \leq x_{i} \\
q_{i}^{+}\left(\xi_{i}-x_{i}\right) & \text { if } & \xi_{i} \geq x_{i}
\end{array}\right.
$$

Therefore, $-\psi_{i}\left(x_{i}, \cdot\right)$ is order preserving with respect to $\precsim_{R_{+}}$when $\xi_{i} \leq x_{i}$ and $\psi_{i}\left(x_{i}, \cdot\right)$ is order preserving with respect to $\precsim_{R_{+}}$when $\xi_{i} \geq x_{i}$. We apply Proposition 7.2 to obtain

$$
-\int_{-\infty}^{x_{i}} \psi_{i}\left(x_{i}, \xi_{i}(\omega)\right) P(d \omega) \leq-\int_{-\infty}^{x_{i}} \psi_{i}\left(x_{i}, \xi_{i}^{L}(\omega)\right) P(d \omega)
$$

and 


$$
\int_{x_{i}}^{\infty} \psi_{i}\left(x_{i}, \xi_{i}^{L}(\omega)\right) P(d \omega) \leq \int_{x_{i}}^{\infty} \psi_{i}\left(x_{i}, \xi_{i}(\omega)\right) P(d \omega)
$$

Adding up these two inequalities and then summing with respect to $i$ yields the assertion involving $\xi^{\mathrm{L}}(\cdot)$. The symmetric inequality with $\xi^{\mathrm{U}}(\cdot)$ is obtained similarly.

7.9 IMPLEMENTATION. The search for random variables $\xi_{i}^{\mathrm{L}}(\cdot)$ and $\xi_{i}^{\mathrm{U}}(\cdot)$ that yield the desired inequalities, can be carried out in terms of the distribution functions $F_{i}^{L}$ and $F_{i}^{U}$ induced by these random variables. Let $F_{i}$ be the distribution function of $\xi_{i}(\cdot)$ with support $\left[\alpha_{i}, \beta_{i}\right]$. The conditions become

$$
\begin{array}{lll}
F_{i}^{L} \geq F_{i} \geq F_{i}^{U} & \text { on } & \left(-\infty, x_{i}\right], \\
F_{i}^{L} \leq F_{i} \leq F_{i}^{U} & \text { on } & {\left[x_{i}, \infty\right) .}
\end{array}
$$

Figure 7.10 gives an example of a discrete distribution $F_{i}^{L}$ that could be used to approximate $\mathrm{F}_{\mathbf{i}}$.

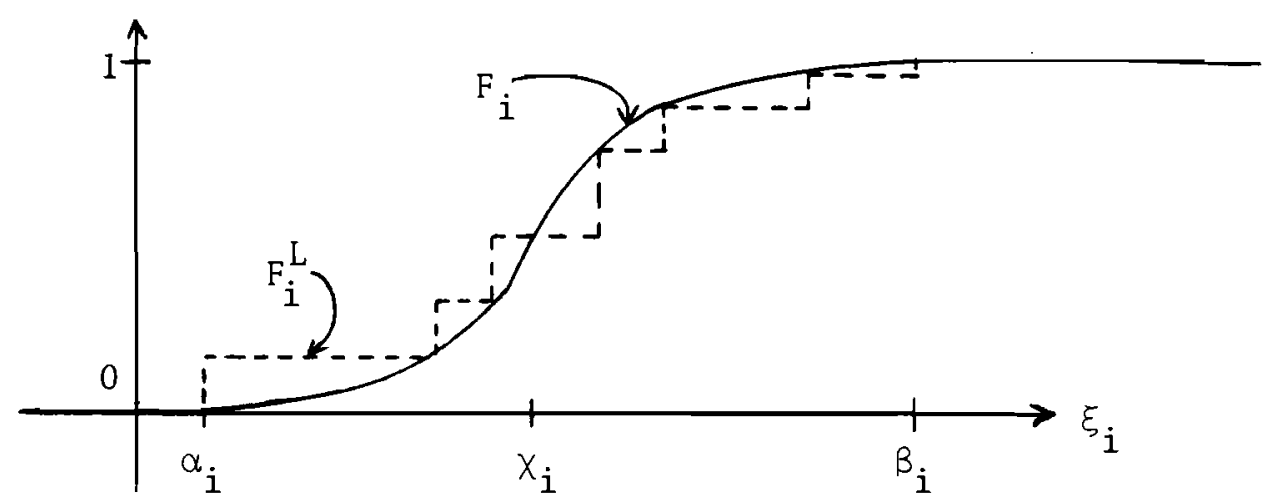

7.10. Figure: Majorizing distribution function $F_{i}^{L}$

As usual, we are only interested in discrete approximations. Our goal is thus to find best discrete approximates that are below or above $F$. Since $F_{i}^{L}=F_{i}=F_{i}^{U}$ at $X_{i}$ we can find the best approximating distribution function that is below 
(or above) $F_{i}$ on each segment $\left(-\infty, X_{i}\right]$ and $\left[X_{i},+\infty\right)$ separately. And since below or above is just a question of reversing signs, we may as well consider the problem at hand in the following framework:

(7.11) find a distribution function $\hat{F}: R \rightarrow[0,1]$

such that $\hat{F} \leq F$,

$\hat{F}$ is piece-wise constant with at most L jumps,

and $\int_{-\infty}^{+\infty}|F(s)-\hat{F}(s)| d s$ is minimized.

We have defined best approximation in terms of the $\ell_{1}$-norm. Recalling that $\hat{\mathrm{F}} \leq \mathrm{F}$, we have

$$
\int|F(s)-\hat{F}(s)| d s=\int F(s) d s-\int \hat{F}(s) d s
$$

and thus we may as well simply maximize $\int \hat{\mathrm{F}}(\mathrm{s}) \mathrm{ds}$ subject to $\hat{\mathrm{F}} \leq \mathrm{F}$. If $z_{1}, \ldots, z_{\mathrm{L}}$ are the points of discontinuity of $\hat{F}$, it is easy to verify that $\hat{F}$ cannot be optimal unless at those points $\hat{F}\left(z_{\ell}\right)=F\left(z_{\ell}\right), \ell=1, \ldots, L$. Taking these observations into account, Problem (7.11) becomes

(7.12) find $\alpha=z_{0} \leq z_{1} \leq z_{2} \leq \cdots \leq z_{L} \leq z_{L+1}=\beta$ such that

$$
\rho(z)=\sum_{\ell=1}^{L}\left(z_{\ell+1}-z_{\ell}\right)\left(F\left(z_{\ell}\right)-F\left(z_{\ell-1}\right)\right) \text { is maximized, }
$$

where $[\alpha, \beta]$ is the support of the distribution function $F$. Note that $\rho$ is not convex. Even with $\mathrm{L}=1$, when $(7.12)$ reads

(7.13) find $z \in[\alpha, \beta]$ such that

$$
\rho(z)=(\beta-z)(F(z)-F(\alpha)) \text { is maximized, }
$$

the solution is not necessarily unique, in fact the solution set may be a disconnected set of points. Assuming that $F$ is twice differentiable with $F$ ' 
denoting the corresponding density, we have that $z^{*}$ is optimal if

$$
\frac{F\left(z^{*}\right)-F(\alpha)}{F^{\prime}\left(z^{*}\right)}=\beta-z^{*} \geq 2 \frac{F^{\prime}\left(z^{*}\right)}{F^{\prime \prime}\left(z^{*}\right)}
$$

which in general has a multiplicity of solutions. To solve (7.12) we propose a heuristic that sequentially adjusts the jump points $z_{1}, \ldots, z_{L}$.

STEP 0 . Pick L points (for example with equal quantiles) in $(\alpha, \beta)$. Set $\ell=0$.

STEP 1. Set $\ell=\ell+1$. Readjust $z_{\ell}$ using the formula:

$$
\begin{aligned}
& z_{\ell}^{\mathrm{n}} \in \underset{z \in\left[z_{\ell-1}^{\mathrm{argmax}}, z_{\ell+1}\right]}{\mathrm{arg}}\left(\mathrm{z}_{\ell+1}-z\right)\left(F(z)-F\left(z_{\ell-1}^{\mathrm{n}}\right)\right) . \\
& \text { Solve using (7.14) exploring the local optima. } \\
& \text { Restart Step } 1 \text { if } \ell<\mathrm{L} \text {; otherwise, go to Step } 2 .
\end{aligned}
$$

STEP 2. Stop if for all $\ell=1, \ldots, L,\left|z_{\ell}^{n} z_{\ell}\right|<\varepsilon$.

Otherwise, return to Step 1 with $z_{\ell}:=z_{\ell}^{n}$ for $\ell=1, \ldots, L$ and $\ell=0$.

This algorithm converges (a monotone increasing sequence bounded above by $\int \mathrm{F}(\mathrm{s}) \mathrm{ds}$ ) but not necessarịly to the optimal solution, this depends on the initial choice of $z_{1}, \ldots, z_{L}$.

An alternative approach to finding the best approximating discrete distribution function is to enter the points $z_{1}, \ldots, z_{L}$ with associated weights. These may correspond to the values of the recourse function, for example. With $v(\cdot)$ as the weighting function, Problem (7.12) becomes 
(7.15) find $\alpha=z_{0} \leq z_{1} \leq \cdots \leq z_{L} \leq z_{L+1}=\beta$ such that

$$
\rho(z)=\sum_{\ell=1}^{L}\left[v\left(z_{\ell+1}\right)-v\left(z_{\ell}\right)\right]\left[F\left(z_{\ell}\right)-F\left(z_{\ell+1}\right)\right] \text { is maximized. }
$$

In the case $L=1$, we have a formula for the optimal $z^{*}$ that corresponds to (7.14), and for the general case the same algorithm, with the obvious modifications, can be used as a heuristic. We could also use generalized programming, as in Section 6 , to solve Problem (7.12) or (7.15). The problem corresponding to (7.25) is then (7.16) find $p_{j} \geq 0, j=1, \ldots, v$ such that

$$
\begin{aligned}
& \sum_{i=1}^{j} p_{i} \leq F\left(z_{j}\right), \quad j=1, \ldots, v \\
& \text { and } \sum_{j=1}^{v} v\left(z_{j}\right) p_{j} \quad \text { is maximized }
\end{aligned}
$$

where $p_{j}=\hat{F}\left(z_{j}\right)-\hat{F}\left(z_{j-1}\right)$. For $v\left(z_{j}\right) \geq 0$, which is usually the case, the optima1 solution is $p_{j}=F\left(z_{j}\right)-F\left(z_{j-1}\right)$. The optimal dual variables associated to (7.16) are defined by

$$
\begin{aligned}
& \sigma_{v}=v\left(z_{v}\right), \\
& \sigma_{j}=v\left(z_{j}\right)-\sum_{\ell=j+1}^{v} \sigma_{\ell} .
\end{aligned}
$$

To add a new point $z_{v+1}$ that generates a new column of (7.16), we need to solve:

$$
\max _{1 \leq j \leq v}\left[\max _{z \in\left[z_{j-1}, z_{j}\right]}\left\{v(z)-\sum_{\ell=j+1}^{v} \sigma_{\ell}\right\}\right] \text {. }
$$

This approach however does not lend itself easily to a fixed upper bound on the number of discontinuities of $\hat{F}$. It could be used to initialize the procedure suggested earlier.

7.18 IMPLEMENTATION. When $\Psi$ is not separable, we can still proceed as in Implementation 7.9 , if we first replace the recourse function by its simple recourse approximate, cf. Application 3.18, at least when seeking an upper bound for $\Psi(X)$. 
8. FURTHER BOUNDS INVOLVING $E_{f}$ OR 2

In this concluding section, we just want to record a number of bounds that require the evaluation of the objective functional $E_{f}$ at some points. The use of these results is thus limited by our capability of evaluating $E_{f}$ (or its gradient) with sufficient accuracy.

To begin, let us simply observe that for all $x \in R^{N}$

$$
\inf E_{f} \leq E_{f}(x)
$$

which gives us a readily available upper bound. Using the subgradient inequality for convex functions we have:

8.2 PROPOSITION. Suppose $\mathrm{x} H \mathrm{f}(\mathrm{x}, \xi): \mathrm{R}^{\mathrm{n}} \rightarrow \mathrm{R} \cup\{+\infty\}$ is a convex function. Then for any pair $\mathrm{x}, \overline{\mathrm{x}}$ in $\mathrm{R}^{\mathrm{n}}$,

$$
E_{f}(x)-E_{f}(\bar{x}) \geq \bar{v} \cdot(x-\bar{x})
$$

with $\bar{v} \in \partial \mathrm{E}_{\mathrm{f}}(\overline{\mathrm{x}})$, provided the set $\partial \mathrm{E}_{\mathrm{f}}(\overline{\mathrm{x}})$ of subgradients of $\mathrm{E}_{\mathrm{f}}$ at $\overline{\mathrm{x}}$ is nonempty. PROOF. Simply observe that $f(\cdot, \xi)$ convex implies that $E_{f}$ is convex which then implies (8.3).

8.4 APPLICATION. Consider the stochastic program with recourse (1.6) with only $\mathrm{h}(\cdot)$ stochastic. Then from [1, Corollary 7.16$]$, we know that with $h=\xi$ :

$$
-E\{\pi(x, \xi)\} T \in \partial Q(x),
$$

where $\pi(x, \cdot): \Omega \rightarrow R^{m_{2}}$ is a measurable function such that

$$
\pi(x, \xi) \in \operatorname{argmax}\{\pi(\xi-T x) \mid \pi W \leq q\} .
$$

Thus, with $f$ as defined by (1.9), we obtain

(8.5) $\quad 2(\hat{x}) \geq 2(x)+E\{\pi(x, \xi(\omega))\} T(x-\hat{x})$. 
8.5 IMPLEMENTATION. Except for some special cases such as stochastic programs with simple recourse, evaluating $2(x)$ or $E\{\pi(x, \xi)\}$ is not feasible, but suppose that $Q^{\mathrm{L}} \leq 2$ where $Q^{\mathrm{L}}$ has been obtained by relying on an approximating measure $\mathrm{P}_{\mathrm{L}}$. Then for any $\hat{x}$ we have that

$$
2(\hat{x}) \geq Q^{L}(\hat{x}) \geq Q^{L}(x)+\int \pi(x, \xi(\omega)) T(x-\hat{x}) P_{L}(d \omega),
$$

with $Q^{L}(x)=\int Q(x, \xi) P_{L}(d \xi)$. The term on the right can now be calculated and gives us a lower bound.

8.6 APPLICATION. Consider the stochastic program with recourse $(1.15)$ with $q(\bullet)$ and $h(\cdot)$ stochastic. As usual

$$
\Psi(X)=E\{\psi(X, \xi(\omega))\},
$$

but let us now also define $p$ as follows:

$$
p(x)=\inf [c x \mid A x=b, T x=x, x \geq 0] .
$$

The stochastic program can then be formulated:

(8.7) find $X \in \mathrm{R}^{\mathrm{m}_{2}}$ such that $\rho(X)+\Psi(X)$ is minimized .

Suppose $\hat{x}$ is a point at which both $\rho$ and $\Psi$ are finite, and suppose $\hat{v} \in \partial \rho(\hat{X})$; the convexity of $\rho$ follows from standard results in parametric linear programming. Let $\stackrel{\vee}{x}$ be such that

$$
-\hat{v} \in \partial \Psi(\stackrel{v}{X})
$$

Assume such a point exists. For any $\chi \in \mathrm{R}^{\mathrm{m}_{2}}$, it follows from the subgradient inequality for convex functions, in particular (8.3), that

and

$$
\rho(x)-\rho(\hat{x}) \geq \hat{v}(x-\hat{x})
$$

$$
\Psi(X)-\Psi(\underline{x}) \geq-\hat{v}(X-\underline{x})
$$

Adding up these two inequalities, we obtain that for all $x$,

$$
\rho(x)+\Psi(x) \geq \rho(\hat{x})+\psi(\stackrel{v}{x})-\hat{v}(\hat{x}-\hat{x})
$$


and hence

(8.8) $\quad \inf (\rho+\Psi) \geq \rho(\hat{x})+\Psi(\tilde{x})-\hat{v}(\hat{x}-\dot{x})$.

We have thus a lower bound for the infimum of the stochastic program.

We note that inequality (8.8) also follows from a duality argument. Assuming that all operations are well-defined:

$$
\begin{aligned}
\inf (\rho+\Psi) & =-(\rho+\Psi) *(0)=-\left(\rho^{*} \square \Psi^{*}\right)(0) \\
& =-\inf _{v}\left(\rho^{*}(v)+\psi^{*}(-v)\right) \\
& \geq-\rho^{*}(v)-\psi^{*}(-v) \quad \text { for all } v,
\end{aligned}
$$

where * denotes conjugacy and $\square$ inf-convolution. Inequality (8.8) now follows from the preceding one with $v=\hat{v}$ and observing that:

$$
\begin{aligned}
\rho^{*}(\hat{v}) & =\hat{v} \hat{\chi}-\rho(\hat{X}) . \\
\Psi^{*}(-\hat{v}) & =-\hat{v} \dot{X}-\Psi(\hat{x}) .
\end{aligned}
$$

This also shows that inequality $(8.8)$ is sharp since

$$
\inf (\rho+\psi)=\sup _{v}\left[-\rho^{*}(v)-\psi *(-v)\right]
$$

8.9 IMPLEMENTATION. Let us illustrate the use of this inequality in the case of a stochastic program with simple recourse with stochastic right-hand sides $\mathrm{h}(\cdot)$. Suppose $\chi^{\mathrm{e}}$ is, for possibly heuristic reasons, believed to be a good guess at the optimal tender (certainty equivalent). Let us now solve the linear program (8.10) find $x \in R_{+}^{n}$, $u^{+} \in R_{+}^{m_{2}}, u^{-} \in R_{+}^{m_{2}}$ such that

$$
\begin{aligned}
& \mathrm{Ax} \quad=\mathrm{b} \text {, } \\
& \mathrm{Tx}+\mathrm{u}^{+}-\mathrm{u}^{-}=\chi^{\mathrm{e}} \text {, and } \\
& \mathrm{cx}+\mathrm{q}^{+} \mathrm{u}^{+}+\mathrm{q}^{-} \mathrm{u}^{-}=z \quad \text { is minimized }
\end{aligned}
$$


where $\mathrm{q}^{+}, \mathrm{q}^{-}$are as usual the recourse costs. Let $\left(\hat{\mathrm{x}}, \hat{\mathrm{u}}^{+}, \hat{\mathrm{u}}^{-}\right)$be the optimal solution, and $(\hat{\sigma}, \hat{\pi})$ the associated simplex multipliers. Then

$$
\hat{\pi} \in \partial \rho(\hat{x})
$$

with $\hat{x}=T \hat{x}$ and $\rho$ as defined in Application 8.6. Moreover, $\hat{\pi} \in\left[-q^{-}, q^{+}\right]$as follows from the optimability conditions, and thus there exists $\stackrel{v}{x}$ such that

$$
-\hat{\pi} \in \partial \Psi(\stackrel{v}{x})
$$

as follows from the formula for subgradients of the recourse function in the simple recourse case [33, Chap.III, Sect.4]. If for $i=1, \ldots, m_{2}, F_{i}$ denotes the distribution function of the random variable $h_{i}(\cdot)$,

$$
\hat{\pi}_{i}=q_{i}^{+}-q_{i} F_{i}\left(\stackrel{v}{x}_{i}\right)
$$

where $q_{i}=q_{i}^{+}+q_{i}^{-}$. With $z^{0}$ the optimal value of the stochastic program $(1.15)$ we have

$$
z^{0} \geq \hat{c x}+\Psi(\stackrel{v}{x})-\hat{\pi}(\hat{x}-\stackrel{v}{x})
$$

Let $\hat{z}=c \hat{x}+\Psi(\hat{X})$ which with the above yields

$$
0 \leq \hat{z}-z^{0} \leq \Psi(\hat{X})-\Psi(\stackrel{v}{X})+\hat{\pi}(\hat{X}-\stackrel{v}{X})
$$

In the case at hand, this becomes [33, Chap.III, Sect.4]

$$
0 \leq \hat{z}-z^{0} \leq \sum_{i=1}^{m_{2}} q_{i} \int_{\substack{x_{i} \\ \hat{x}_{i}}}^{\hat{x}_{i}}\left(\zeta-\hat{x}_{i}\right) d F_{i}(\zeta),
$$

which is known as Wizlioms' inequality. Let us point out that the path followed to obtain this last inequality, using $(8.8)$ is quite different from the original proof of Williams [34] and should clarify the underpinnings of this result. 


\section{REFERENCES}

[1] R. Wets, Stochastic programs with fixed recourse: The equivalent deterministic program, SIAM Review, 16(1974), 309-339.

[2] R. Wets, Convergence of convex functions, variational inequalities and convex optimization problems, in Variational Inequalities and Complementarity Problems, eds. R. Cottle, F. Giannessi, and J.-L. Lions, J. Wiley \& Sons, New York, 1980, 375-403.

[3] H. Attouch, Variational Convergence for Functions and Operators, Research Notes in Mathematics Pitman, London, to appear.

[4] H. Attouch and R. Wets, Approximation and convergence in nonlinear optimization, in NonZinear Programming 4, eds. O. Mangasarian, R. Meyer and S. Robinson, Academic Press, New York, 1981, 367-394.

[5] H. Attouch and R. Wets, A convergence theory for saddle functions, Trans. Amer. Mat. Soc. (1983).

[6] R. Wets, Stochastic programming: solution techniques and approximation schemes, in Mathematical Programing: The State-of-the-Art 1982, eds. A. Bachem, M. Grötschel and B. Korte, Springer-Verlag, Ber1in, 1983, 566-603.

[7] R. Wets, Modeling and solution strategies for unconstrained stochastic optimization problems, Annals of Operations Research 1(1984); also IIASA Working Paper WP-83-36, Laxenburg, Austria.

[8] J. Wang, Distribution sensitivity analysis for stochastic programs with recourse, IIASA Working Paper WP-82-133, Laxenburg, Austria.

[9] R. Wets, Solving stochastic programs with simple recourse II, in Proceed. 1975 Conference on Information Sciences and Systems, The Johns Hopkins Univsersity, Baltimore, 1975.

[10] J. Birge, The value of the stochastic solution in stochastic linear programs with fixed recourse, Mathematical Programing 24(1982), 314-325.

[11] R.T. Rockafellar and R. Wets, Stochastic convex programming: basic duality, Pacific J. Mathem. 62(1976), 173-195.

[12] A. Madansky, Inequalities for stochastic linear programming problems, Management Science, 6(1960), 197-204.

[13] P. Kall, Computational methods for solving two-stage stochastic linear programming problems, Z. Angew. Math. Phys. 30(1979), 261-271.

[14] C. Huang, I. Vertinsky and W. Ziemba, Sharp bounds on the value of perfect information, Operations Research 25(1977), 315-325.

[15] M. Perlman, Jensen's inequality for a convex vector-valued function in an infinite dimensional space, J. Multivariate Analysis, 4(1974), 52-65.

[16] J. Birge and R. Wets, Approximations and error bounds in stochastic programming, in Proceedings 1982 Nebraska Conference on Inequalities in Statistics and Probability, ed. Y. Tong, IMS Publications; also IIASA Working Paper 1983, Laxenburg, Austria. 
[17] P. Kal1, Lecture at IIASA, May 1983.

[18] P. Kall and D. Stoyan, Solving stochastic programming problems with recourse including error bounds, Math. Operationsforsch. Statist., Ser. Optimization, $13(1982), 431-447$.

[19] C. Huang, W. Ziemba and A. Ben-Ta1, Bounds on the expectation of a convex function of a randon variable: with applications to stochastic programming, Operations Research, 25(1977), 315-325.

[20] M. Krein and A. Nude1man, The Markov Moment Problem and Extremal Problems, Translation Mathem. Monographs 50, Amer. Math. Soc., Providence, 1977.

[21] A. Karr, Extreme points of certain sets of probability measures, with applications, Mathematics of Operations Research, 8(1983), 74-85.

[22] J. Zackgva, On minimax solutions of stochastic linear programming problems, Cas. pest. mat. 91(1966), 423-430.

[23] J. Dupacova, Minimax stochastic programs with nonconvex nonseparable penalty functions, in Coll. Math. Sci. J. Bólyai 12, Progress in Operations Research Eger 1974. North-Holland 1976, 303-316.

[24] J. Dupačova, Minimax approach to stochastic 1 inear programming and the moment problem, ZAMM 58(1978), T466-467; see also EMO 13(1977), 279-307.

[25] G. Dantzig, Linear Programing and Extensions, Princeton University Press, Princeton, 1963.

[26] Y. Ermoliev, A. Gaivoronsky and C. Nedeva, Stochastic optimization problems with partially known distribution functions, IIASA Working Paper, 1983.

[27] R. Wets, Gmundlagen Konvexer Optimiemung, Lecture Notes in Economics and Mathematical Systems 137, Springer-Verlag, Berlin, 1976.

[28] T. Cipra, Class of unimodal distributions and its transformations, Cas. pest. mat. $103(1978), 17-26$.

[29] A. Cabot, Variations on a cutting plane method for solving concave minimization problems with linear constraints, Naval Research Logistics Quarterly, 21 (1974), 265-279.

[30] H. Don, Bounds on the expected value of a convex function in a sample-based class of probability measures, IIASA Notes, August 1983.

[31] V. Garcia-Palomares and A. Restuccia, Global quadratic algorithm for solving a system of mixed euqalities and inequalities, Mathematical Programing $21(1981), 290-300$.

[32] A. Marshall and I. Olkin, Inequalities: Theory of Majorization and its Applications, Academic Press, New York, 1979.

[33] P. Kall, Stochastic Linear Programing, Springer-Verlag, Berlin, 1976.

[34] A. Williams, Approximation formulas for stochastic linear programming, J. SIAM App Z. Math., 14(1966), 668-677. 